580.5

FB

V. 2

1900-1916

cop, 2

Inc. 
$\therefore$ 
Field Columbian Museum.

Publication io6.

Botanical Series.

Vol. II, No. 3 .

\section{PRÆNUNCIÆ BAHAMENSES-I.}

CONTRIBUTIONS TO A FLORA OF THE BAHAMIAN ARCHIPELAGO.

BY

Charles Frederick Millspaugh, M.D.

Curator Department of Botany.

Chicago, U. S. A.

February, 1906. 


\title{
BOTANICAL SERIES.
}

\author{
ACTUAL DATES OF ISSUE.
}

\section{Publication}

Number.

Bot. Ser.

Title

Date of Issue

4 Vol. I No. I Flora of Yucatan.

Sept. 20,1895

9 Vol. I No. 2 Flora of West Virginia.

Mar. 21,1896

I 5 Vol. I No. 3 Contrib. Fl. Yucatan II. Mar. I, 1897

25 Vol. I No. 4 Contrib. Fl. Yucatan III. Mar. 7, 1898

39 Vol. I No. 5 Higinbothamia, etc.

Sept. 9, I 89

43 Vol. II No. I Plantae Utowanæ.

Apr. 20, 1900

48 Vol. I No. 6 Pl. Ins. Ananas.

Sept. 7,1900

50 Vol. II No. 2 Pl: Uto. Suppl.

Sépt. 7, r 900

68 Vol. I No. 7 Fl. St. Croix.

Dec. 16,1902

69 Vol. III No. I Pl. Yuc. Fasc. I.

92 Vol. III No. 2 Pl. Yuc. Fasc. II.

Apr. 6, 1903

Apr. 26, 1904

Volume I Is closed and indexed.

Volume II Is still open. Reserved for papers on Antillea Botany.

Volume III Is still open. It is reserved for the sequence fascicles of Plantae Yucatanae until that Flora completed. 
Field Columbian Museum.

Publication io6.

Botanical Series:

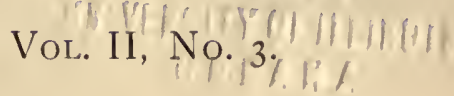

\section{PRÆNUNCIÆ BAHAMENSES-I.}

CONTRIBUTIONS TO A FLORA OF THE BAHAMIAN ARCHIPELAGO.

BY

Charles Frederick Millspaugh, M.D.

Curator Department of Botany.

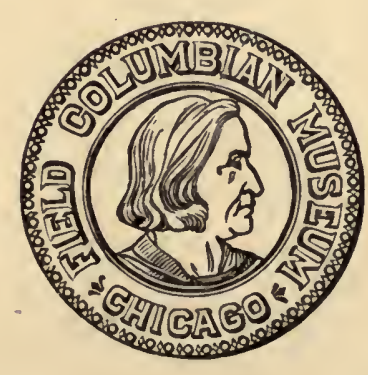

Chicago, U. S. A.

February, 1906. 
18)18

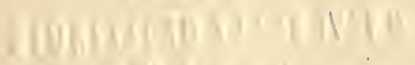

. . W 


\title{
PRÆNUNCIÆ BAHAMENSES - I.
}

\author{
CONTRIBUTIONS TO A FLORA OF THE BAHAMIAN ARCHIPELAGO.
}

CHARLES F. MILLSPAUGH, M.D.

During field and herbarium studies of the flora of the Greater Antillean Islands, conducted by Dr. N. L. Britton, Director of the New York Botanical Garden, and the Author, each came to the conclusion that a thorough investigation of the Bahamian Archipelago would throw much desired light upon many problems of plant distribution and mutation, and offer a probable solution of the question of the age of these interesting islands.

A joint investigation of such collections as had been previously made on the archipelago proved them to be in inany cases meagre in material, scrappy in character, or from islands too widely separated.to afford even a partial solution of the problems presented. They therefore decided to solicit the consent of those botanists representing the Government of Great Britain, to which these islands belong, and the support of the institutions they. themselves represent, in view of organizing a thorough exploration of the islands included in the archipelago. This being in both instances unhesitatingly given, three expeditions were equipped for immediate field work and others planned, to cover within the next two years, all the islands included.

Up to the present writing, the survey thus organized has covered the following groups:-Great Bahama, the Abacos, the Isaacs, the Berry Islands, the Bimini Islands, the New Providence area, the Exuma Chain, the Inaguas and Grand Turk. In these, a large amount of information and material has been accumulated by Dr. and Mrs. Britton, Mr. Nash, Mr. Brace, and the Author. In accomplishing this, not only have the usual lines of communication been utilized, but special vessels have been equipped and commissioned. 
The following material (over 7,000 sheets), resulting from this survey, or secured by exchange, gift, loan, or purchase, is deposited in duplicate in both institutions, where it is being studied by the co-workers, who are also taking occasion at various intervals to consult the collections of Catesby, Eggers, Hjalmarsson, Brace, Daniell, Herrick, Allen, Barbour \& Bryant, and others, deposited in European and American herbaria. In addition, a complete series of the orchids, cacti, tillandsias, and other difficult groups is being collected alive and studied in the conservatories of the Garden.

\section{COLLECTÓRS.}

Brace - Mr. Louis J. K. Brace began his collections in the Bahamas in I 875. He worked principally on New Providence until i 880, but also secured material on Fortune Island or Long Cay, and Andros. His work resulted in about 525 numbers, which were sent to Kew Gardens. A duplicate set is still in his possession. He began field work under the present survey.in September, igo4 (see Britton \& Brace), and later in the same year was commissioned by the Garden to make an exploration of the Abaco Islands, where he collected as follows: Green Turtle Cay (I475-I5I5), Allen's Cay (1526-54), Spanish

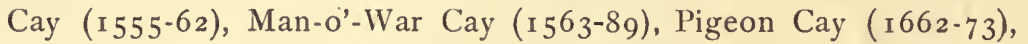
Elbow Cay (I688-1712), and on the island of Abaco at Butler Bay (1516-25), Marsh Harbor (г590-166г, I7г3-53, I 787-1846), Great Cistern (1674-87, I754-86), Cherokee Sound (I847-9, I898-г980, I 999, 2080-84, 2086), Eight Mile Bay ( I 850-97), opposite Cherokee Settlement (I98I-98, 2064-79), Old Kerr's Point (2000-2036), and California road (2037-63), a total of 6 I I numbers. Following this expedition he continued work on New Providence in localities from which, from time to time, it became necessary to have more material of previously secured species.

In June, I905, a commission was given him by both the Garden and this Museum to continue, on Great Bahama, the work already undertaken there. (See Britton \& Millspaugh.) On this exploration he collected at Eight Mile Rocks (3633-5, 3671-3738), Deadman's Reef (363r-2), and the western extremity of the island $(3484-3630$, 3636-57). He also collected Garden Cay (3658-70), and visited (during his sailings to and from Great Bahama) North Bimini (346r-75, 3479-83), South Bimini (3476-8), North Cat Cay (3739$54)$, and Andros Island ( $\left.3755^{-6}\right)$, and after returning to New Providence collected Nos. 3893-39 I 8.

Britton - Dr. N. L. Britton collected on New Providence in April, I 904, in the neighborhood of Nassau, South Side Beach, Blue Hills, Far- 
ringdon Road, West Bay Street Road, Cunningham Road, Lake Cunningham, and Old Fort. In this preliminary investigation, in company with the Author (see Millsp.), he collected $5^{8}$ numbers, (I-I 58). An account of the field work is published in the Journal of the New York Botanical Garden 5: г $29^{-1} 3^{6 .}$

See also Brittor: \& Brace, and Britton \& Millspaugh.

$B$. \& Br. - Britton \& Brace-Dr. N. I. Britton, Mrs. E. G. Britton, and Mr. Louis J. K. Brace collected together on New Providence (Nos. 159-328, 36r-872), and Hog Island (329-360), in September and October, 1904. They ranged New Providence with great thoroughness, penetrating to the coast region north, east, south, and west, and to the coppices, pine barrens, and openings in the neighborhood of Nassau, Fort Montague, Farringdon Road, Swingate, Maidenhead, Tea House, Race Course, Delaport, Fox Hills, Village Road, Mt. Vernon, Rifle Range, Harold Road, Carmichael, Adelaide, Southwest Landing, Miller's, Blue Hills, Soldiers Road, Lake Cunningham, Winton, Old Fort, Waterloo, Clifton, Mt. Pleasant, Fort Charlotte, Gambier, Killarney, Bonefish Pond, and Seven Hills. The commoner things were not taken, the attempt being made to secure only such species as were previously unfamiliar or those which had only been gathered when out of character. This survey resulted in 7 I 4 particularly interesting plants embracing the series of numbers indicated above.

An account of this expedition is published in the Journal of the New York Botanical Garden 5: 20I-209.

B. \& $M$. - Britton \& Millspaugh - Drs. N. L. Britton and C. F. Milispaugh, accompanied by Mr. M. A. Howe, began an exploration of the Berry Islands, Great Bahama, and the Exuma Chain, in January, I905. A schooner was chartered and equipped at Nassau, where, after a day's delay on account of heavy weather (during which collecting was done in the scrublands and coppices south of Nassau (2085-2II0), the expedition began at Rose Island (2III-2I66), and Hog Island $(2167-2176)$. The first island of the Berry Group visited was Whale Cay (2177-220I), followed by Frozen Cay (22022224), Little Harbor Cay (2225-2254), Great Sturrup Cay (22552285), Goat Cay (2286-2301), Lignum Vitæ Cay (2302-2332), and Great Harbor Cay (2333-2354). The exploration began on Great Bahama Island at Eight Mile Rocks $\left(2355^{-2} 59^{6}\right)$, and was continued at Barnett's Point (2597-2716), and Golden Grove (2717-274I). Returning to Nassau, a fresh start was made to the Exuma Chain, of which the following islands were diligently searched: Ship Channel 
Cay (2742-2767), Cay north of Wide Opening (2768-2804), Cave Cay (2805-2836), Little Galiot (2837-2850), Great Galiot (285I. 2860), Great Guana (2861-2922), Great Exuma (2923-305I, 30733r $4 \mathrm{r}$ ), and Stocking Island (3052-3072).

On Great Exuma the regions worked were the scrub lands and coppices lying west of Georgetown; Hayne's Road from the east to the west shores across the island; and the Rolletown scrub lands and coppices. The total series of this exploration is 1057 sheets.

An account of this expedition may be found in the Journal of the New York Botanical Garden 6: 77-85.

Coker - Dr. William C. Coker, botanist of the Bahamian expedition of the Geographical Society of Baltimore, assisted by Messrs. C. A. Shore and F. M. Hanes, collected in the summer of 1903 on the following islands: New Providence (I-I93, 247-305, 55I), Andros Island and Little and Great Mangrove Cays (194-234), Green Cay (235-246), George's Island (306-3I 7), Eleuthera (3I8-42 I), Cat Island (422-437), Ruin Cay (438-457), Watlings Island (458-49I, $521,528)$, Long Island $(492-520,524)$, Water Cay $(523,525)$, A baco $\left(55^{8}-568,575^{-6}\right)$, and Elbow Cay $(569-574)$. This collection was deposited in the herbarium of the New York Botanical Garden, and forms the basis of his "Vegetation of the Bahama Islands," in Shattuck's "The Bahama Islands." Owing to the insufficiency of the material secured many phanerogams therein published are based upon provisional determination only. The cryptogams exist under a separate series of numbers.

Cooper - Dr. William Cooper paid a visit to New Providence in 1859 for the purpose of making dredgings for zoölogical material. While there he collected in the neighborhood of Nassau about roo plants for his friend, Dr. John Torrey. - The prime set of these plants is now in the herbarium of the New York Botanical Garden. See Bull. Torrey Club i $7: 190$.

Curtiss - Mr. A. H. Curtiss collected his first series of "West. Indian Plants" in April, 1903, in the neighborhood of Nassau, New Providence. This series comprises numbers I-2II, of which the prime set, with the unnumbered unicates, is deposited in the herbarium of the New York Botanical Garden, and the first distributed set in the herbarium of this Museum.

Earle - Prof. F. S. Earle collected during one day only, on New Providence (Nos. I-79), while on his way to Cuba, in March, I903. His plants, largely from the vicinity of Nassau, are in the herbarium of the Garden. 
E. G. B. - Mrs. N. L. Britton, often accompanied by Mr. Brace, visited, in April and May, 1905, all those stations on New Providence previously collected by Britton \& Brace, at which it had become desirable to secure additional material of interesting plants or characters missing on previous notable species. In addition to achieving marked success in this undertaking, she secured many other plants of particular interest. Her survey resulted in 3 Io sheets (Nos. 3141-3460), and is mentioned in the Journal of the New York Botanical Garden 5:129-136. See also Britton \& Brace.

Hitchcock-Dr. J. T. Rothrock and Prof. Albert S. Hitchcock collected in the Bahamas in November and December, 1890 . The collections were largely made by Prof. Hitchcock on New Providence, Eleuthera, Cat, Watlings, Crooked, Fortune, and Inagua, and form the basis of his "Plants collected in the Bahamas," etc. The. material collected was deposited in the herbarium of the Missouri Botanical Garden, from which, through the kindness of the director, Dr. William Trelease, the author has been allowed to reassemble the sheets. The collection, embracing about 600 sheets, is at this writing a loan to the Field Columbian Museum.

Hoze'e - Dr. Marshall A. Howe, algologist of the New York Botanical Garden, has collected extensively the sea vegetation of the Bahamas, accompanying Dr. Britton and the author on their first trip, and later, Britton \& Millspaugh. He also accompanied the author upun the Bimini explorations. See Journal New York Botanical Garden $5: 129-\bar{I}_{3} 6$ and $6: 77 \cdot 85$.

Millsp. - C. F. Millspangh-the author began his collections among the islands on New Providence, in April, I904, in company with Dr. Britton (see Britton), visiting substantially the same stations, where, as his collecting was to extend to other islands, each kept his individual series of numerals. He collected at the following localities: Blue Hills (2048-2100), South Shore (210I-2I5I and 2262-2277), West Bay Street road (2152-2I78), pine barrens and hammock lands along Lake Cunningham road (2I79-22I7), region of Lake Cunningham (2218-2244), Farringdon Road (2245-7), Swingate (22485I), Old Fort (2252-4), and Waterloo (2255-6I). Then hiring and commissioning a sloop, he sailed in company with Dr. M. A. Howe(see Howe) for an investigation of the Bimini Islands, collecting at the following stations: West End Bight, New Providence (2278-2293), Joulter's Cays (2294-2304), Gun Cay (2305-2328), North Cat Cay (2329-2347, 24I5), South Bimini (2348-2368, 2379-85, 2387-98, 
2406-24I4), North Bimini (2369-78, 2386, 2399-2405), South Cat Cay, (24I6-244I).* Again in January, I905, he collected in the following localities while awaiting the arrival of Dr. Britton (see Britton \& Millspaugh): Blue Hills road (2442-4, 246r-8r), Silver Cay (2445-2460), and South Side, Soldiers Road, Fort Montague and Fort Fincastle (2482-2502). Following immediately upon this, see Britton \& Millspaugh.

N. \& T. - Nash \& Taylor-Messrs. Geo. V. Nash and Norman Taylor, of the New York Botanical Garden, were commissioned by the Garden, in October, I904, to investigate the Inaguas. They made an exhaustive survey of the islands, collecting on Inagua 482 numbers (874-II38 and I258-I474), on Sheep Cay 23 numbers (I I39-II61), and on Little Inagua 96 numbers (I $62-1257$ ). In September, I 905 , on their return from an exploration in Haiti, they spent a week on Grand Turk Island, collecting all such plants as were noted by them at that season, 136 numbers $(3757-3892$.) Accounts of these expeditions may be found in the Journal of the New York Botanical Garden 6:I-I9 and I89-I9I.

Northrop - Mrs. Alice (Rich) Northrop, accompanied by her husband, Prof. John I. Northrop, made, in 1890 , a large collection of the plants of New Providence (Nos. I-239, 280-33I), Rose Island (25I), Salt Cay (240-244, 27 I-279), Hog Island (245-8, 252-70), and Andros (332-758). These plants formed the basis of Mrs. Northrop's "Flora of New Providence and Andros." They are now deposited in equal sets in the herbaria of this Museum and the Garden.

Wight-Mr. Alex. E. Wight, under the patronage of the Gray Herbarium, of Cambridge, Mass., made a Bahamian collection of 275 numbers from March to May, i905. He collected on New Providence and Hog Island (Nos. I 226 and 271-275), and on Andros (227270). Theresulting plants are being determined at the Gray Herbarium, where the initial set will be retained and the duplicate sets distributed to this Museum, the New York Botanical Garden, and others. Prof. B. L. Robinson has kindly communicated, in advance, to the author all those numbers falling within the natural orders treated in this Prænuncia.

*An account of this expedition may be found in the Journal of the New York Botanical Garden 5:129-136. 


\section{Islands Represented in the Collections.}

Abaco - Brace, Coker.

Allen's Cay (Abaco) - Brace.

Andros - Brace, Coker, Northrop, Wight.

Bimini, North - Brace, Howe, Millspaugh.

Bimini, South - Brace, Howe, Millspaugh.

Cat Cay, North (Biminis) - Brace, Howe, Millspaugh.

Cat Cay, South (Biminis) - Howe, Millspaugh.

Cat Island - Hitchcock.

Cave Cay (Exuma Chain) - Britton \& Millspaugh, Howe.

Crooked Island - Hitchcock.

Eleuthera - Coker, Hitchcock.

Elbow Cay (Abaco) - Brace, Coker.

Exuma-Britton \&o Millspaugh, Howe.

Fortune Island-Brace, Hitchcock.

Frozen Cay (Berry Is.) - Britton \& Millspaugh, Howe.

Galiot Cay, Great (Exuma Chain) - Britton \& Millspaugh, Howe.

Galiot Cay, Little (Exuma Chain) - Britton \& Millspaugh, Howe.

Garden Cay (Gt. Bahama) - Brace.

George's Isl. (Eleuthera) - Coker.

Goat Cay (Berry Is.) - Britton \&o Millspaugh.

Great Bahama Island - Brace, Britton of Millspaugh, Howe..

Green Cay - Coker.

Green Turtle Cay (Abaco) - Brace.

Guana, Great (Exuma Chain) - Britton \& Millspaugh, Howe.

Gun Cay (Biminis) - Howe, Millspaugh.

Harbor Cay, Great (Berry Isl.) - Britton \& Millspaugh, Howe.

Harbor Cay, Little (Berry Isl.) - Britton \& Miltspaugh, Howe.

Hog Island (N. Providence) - Brace, Britton, N. L., Britton, E. G.,

Britton \&o Brace, Britton \&o Millspaugh, Northrop, Wight.

Inagua-Hitchcock, Nash \&o Taylor.

Inagua, Little - Nash \& Taylor.

Isaac, Great - Brace.

Joulter's Cays (Andros) - Howe, Millspaugh.

Lignum Vitæ Cay (Berry Is.) - Britton \& Millspaugh, Howe.

Long Cay see Fortune Island.

Long Island - Coker.

Mangrove Cay, Little (Andros) - Cokęr.

Mangrove Cay (Andros) - Coker. 
Man o'War Cay (Abaco) - Brace.

New Providence - Brace, Britton, N. L., Britton, E. G., Britton \& Brace, Britton \&o Millspaugh, Coker, Cooper, Curtiss, Earle, Hitchcock, Howe, Millspaugh, Northrop, Wight.

No Harbor Cay (Rose Isl.) - Britton \& Millspaugh.

Pigeon Cay (Abaco) - Brace.

Rose Island (N. Providence) - Britton \& Millspaugh, Howe, Northrop. Rum Cay - Coker.

Salt Cay (N. Providence) -- Northrop.

Sheep Cay (Inagua) - Nash \& Taylor.

Ship Channel Cay (Exuma Chain) - Britton \& Millspaugh, Howe.

Silver Cay (N. Providence) - Howe, Millspaugh.

Spanish Cay (Abaco) - Brace.

Stocking Island (Exuma Chain) - Britton \& Millspaugh, Howe.

Sturrup Cay, Great (Berry Isl.) - Britton \& Millspaugh, Howe.

Turk Island, Grand - Nash \& Taylor.

Water Cay (Cay Sal Bank) - Coker.

Watlings Island - Coker, Hitchoock.

Whale Cay (Berry Islands) - Britton \& Millspaugh, Howe.

Wide Opening, Cay N. of (Exuma Chain)-Britton \& Millspaugh, Howe.

Critical investigation of the above collections has so far resulted in the following publications:

"A New Waltheria from the Bahamas". N. L. Britton in Torreya 3: 105 (July 25, I903).

"Notes on Bahaman Algæ" Marshall A. Howe in Bull. Torr. Club, 3I: 93-100 (Feb. II, I904).

"Savia Bahamensis" N. L. Britton in Torreya 4: I04 (Nov. 2 I, I 904). "A new Bahaman Euphorbia" C. F. Millspaugh in Torreya 4: I72 (Nov. 21, I904).

"Notes on the Flora of the Bahamas" N. L. Britton before the Torry*Botanical Club. Digest in Torreya 4: 190 (Dec. 30, I904). "On Pisonia obtusata and its Allies" N. L. Britton in Bull. Torr. Club 3r: 6rir-6r5 (Jan. 9, r905).

"Contributions to the Flora of the Bahama Islands - I" N. L. Britton in Bull. New York' Bot. Gard., 3: 44I-453 (Feb. 7, 1905).

"The Polyporaceæ of North America-X." W. A. Murrill in Bull. Torr. Club 32: 9r. Agaricus deplanatus (Fr.) Murr. (March 22, I 905$)$. 
"Phycological Studies - J" Marshall A. Howe in Bull. Torr. Club 32: $24 \mathrm{I}-252$ (May 6, I905).

"Contributions to the Flora of the Bahama Islands - II" N. L. Britton in Bull. New York Bot. Gard. 4: I I 5-I 27 (Aug. 24, I 905).

"Phycological Studies - II" Marshall A. Howe in Bull. Torr. Club 31:563-586 (Dec. 6, I905). 


\section{AMARANTHACEÆ， EUPHORBIACEÆ， RUBIACEÆ, VERBENACEÆ, AND SOLANUM DIDYMACANTHUM.}

\section{AMARANTHACE E.}

Amaranthus crassipes Schlecht.

Waste grounds infrequent:

New Providence - Fort Charlotte, B. \& Br. 778 .

Amaranthus emarginatus Salzm.

In cultivated grounds:

New Providence - Nassau, B. \& Br. 794.

Not before known from the Bahamas. There appears to be no doubt as to the validity of this species. It comes up from Brazil through Guadeloupe (Duss 4067 ) to the Bahamas.

Amaranthus hybridus Linn.

Waste grounds about dwellings:

Fortune Island - Hitchcock (A paniculatus).

Amaranthus polygonoides Linn.

Sandy waste places:

Eleuthera - Governor's Harbor, Hitchcock.

Inagua $-N$. \& $T$. 1079 .

\section{Amaranthus spinosus Linn.}

Waste grounds:

New Providence - Nassau, Hitchcock;"Brace 204.

Amaranthus tristis Linn.

Waste grounds near habitations:

Abaco - Cherokee Sound, Brace 1922.

New Providence - Grants Town, B. \& Br. 792; Soldiers Road, Coker 264 .

Great Guana Cay - B. \& M. 2910.

Not before known from the Bahamas. 


\section{Amaranthus viridis Linn.}

Waste grounds near dwellings:

Great Bahama - Eight Mile Rocks Settlement, B. \& M. 2429.

New Providence - Nassau, $B$. \& Br. 785; Brace 9, 206; Curtiss sine num.

Not before known from the Bahamas.

Achyranthes indica (L.) Mill.

A. aspera obtusifolia (Lam) Griseb. A weed in waste places and cultivated soils:

Abaco - Cherokee Sound, Brace 1921.

New Providence - Nassau, Earle 23; B. \&.M. 2086; Hitchcock.

Exuma - Georgetown, B. \& M. 2991.

Alternanthera maritima St. Hil.

In maritime sands on the upper beach line, often within wash of the waves:

Great Bahama - Barnett's Point, B. S M. 2620; near Dead Man's Reef, Brace 3632 .

Frozen Cay - B. E M. 2205.

South Bimini-Millsp. 2353, 2410.

New Providence - Southwest Bay, B. \& Br. 478 .

Alternanthera paronychioides St. Hil.

In sand, especially where trodden down:

Abaco - Cherokee Sound, Brace 1925.

Great Bahama - Eight Mile Rocks, B. \& M. 2367, 2412, 2419; Road to

Dead Man's Reef, Brace 3623.

New Providence - near Nassau, Hitchcock (A repens); Northrop 197; $B$. \& Br. 263; Curtiss 161; Wight 121.

St. George's Cay - Coker 313.

Inagua - N. \& T.979.

Lithophila muscoides Sw.

Usually in trodden sands of roadside paths:

Great Bahama - Eight Mile Rocks, B. \& M. 2573.

New Providence - interior of Old Fort, Millsp. 2253; Nassau, Curtiss 60; Northrop 150.

Inagua - Hitchcock (Alternanthera muscoides); N. \& T. 892.

Grand Turk Island - N. \& T. 3786.

Lithophila vermicularis (L.) Uline.

On maritime rocks and beach dunes:

Abaco - Butler Bay, Brace 1514.

Great Bahama - Barnett's Point, $B$. \& $M$. 
Great Sturrup Cay $-B$. \& $M$.

Frozen Cay $-B$. \& $M$.

South Bimini - Millsp. 2409.

Little Mangrove Cay - Coker 201.

Rose Island - $B$. \& $M$.

New Providence - Southwest Bay, B. \& Br. 459; Swingate,Millsp. 225I;

Nassau neighborhood, Coker 37; Cooper sine num.; Northrop 147; Wight IO.

Eleuthera - Governor's Harbor, Hitchcock (Philoxerus vermicularis).

Exuma - rocks of West Beach, B. \& $M$.

Watlings Island - Coker 476 .

\section{Iresine keyensis sp. nov.}

Caule lignoso erecto ramoso glabro, foliis petiolatis anguste lanceolatis vel oblongo-lanceolatis rotundo-obtusis submucronatis basi ad petiolam utrubique decurrentibus, margine integris subrevolutis pagina utrinque minute pustulosis, paniculis elongatis tenuiter ramosis laxis subaphyllis, rachi glabris, floribus albis paleaceis, calyce bracteas laterales medio superante, sepalis obtusiusculis enerviis glabris. Semen obeso-lenticularis.

Plants strictly erect $4.5-6$. ० dm. Leaves $4 \mathrm{~cm}$. $\times 6$ min., $9 \times \mathrm{I} .5 \mathrm{~cm}$. to $6 \times 2 \mathrm{~cm}$. petiole about one-quarter the length of the blade, panicles I $-2 \mathrm{dm}$. the ultimate branchlets often up to $4 \mathrm{~cm}$. long, very delicate, almost capillary. Seeds dark-red, smooth and polished $.5 \times .5 \mathrm{~mm}$.

On maritime rocks:

Great Bahama - Eỉght Mile Rocks, B. \& M. 2485.

Great Sturrup Cay $-B$. \& $M$.

Little Harbor Cay - B. \& $M$.

Lignum Vitae Cay - where it forms a strong character plant, $B$. \& $M$.

Goat Cay - B. \& M. 2289.

Frozen Cay $-B$. \& $M$.

Whale Cay - B. \& M. 2179 type.

South Bimini - Millsp. 2390.

South Cat Cay - Millsp. 2437.

Andros - Calabash Cay, Stafford Creek, Northrop $362 a$ (I. paniculata).

Here called "NEwBURN WEED."

Mangrove Cay - Bryant 11 .

Silver Cay - Millsp. 2450.

New Providence - Southwest Bay, B. \& Br. 480; neighborhood of Nassau, Brace 424; Hitchcock (I.celosioides)

Eleuthera - Governor's Harbor, Hitchcock (1. celosioides).

Great Galiot Cay - B. \& M M. 2854.

Cave Cay -B. \& M. 2820. 
Exuma - West Beach, B. \& $M$.

Cat Cay - Port Howe, Hitchcock (I. celosioides).

Watlings Island - Hitchcock (I. celosioides).

Iresine inaguensis sp. nov.

Caule ad basin lignoso erecto ramosissimo glabro. Foliis sessilis oppositis vel opposito - fasciculatis, glabris, anguste linearis obtusis margine integris; paniculis elongatis, terminalis densus et tenuiter ramosissimis subaphyllis, ramis densis, spiculis oppositis vel alternis, sessilibus, tenuis, rachi glabris, floribus sessilis paleaceis, calyce bracteas laterales paulo superante, sepalis acutiusculis deltoideis in sicco carinatis; utriculus glaber, semen reniforme, obesum, nitidum, fusco-nigrum.

Plants erect $6-9 \mathrm{dm}$. compactly myriad branched, branches tenuous, internodes $5-8 \mathrm{~cm}$. long, leaves $3-6 \mathrm{~cm}$. long, I $\mathrm{mm}$. wide or slightly larger. Seeds $.6 \times .5 \mathrm{~mm}$.

Sheep Cay - Inagua, Oct. 18, 1904, Nash \& Taylor 1139 type.

Inagua - Salt Pond Hill, $N$. \& T. 929. This with almost capilliform leaves.

\section{EUPHORBIACEÆ}

Savia bahamensis Britton.

Rocky places in coppices and pine barrens :

Abaco - Marsh Harbor, Brace 1644, 1723, 1609, 1638, and Cherokee

Sound, Brace 1965, 1970.

Great Bahama - Eight Mile Rocks, B. \& $M$. 2407; West End, Brace $3504,3501,3640$.

Andros - Fresh Creek, Northrop 610 (S. erythroxyloides), where it is known as "MaIden Bush."

New Providence-Brace 345, 3906; B. \& Br. 670; Coker 157, 160; Coppice on West Bay Street Road, Britton 84 type; and Millsp. 2163 from the same bush.

Hog Island - B. \& Br. 438 .

Wide Opening, Cay north of $-B$. \& $M .2772$.

Exuma - near Georgetown, B. \& M. 2959.

Inagua $-N$. \& $T .1439$.

Securinega acidothamnus (Griseb.) Muell.

In maritime coppices and scrub lands:

New Providence - at Southwest Landing, B. \& Br. 475; E. G. B. 3332.

Mangrove Cay - Coker 225.

Exuma - near Georgetown. B. \& M. 2958.

(Phyllanthus angustifolius Gard. \& Br.

This species of Swartz, reported by Gardner \& Brace in Proc. Acad. Nat. Sci. Phila. I889: 405, has not been collected by Mr. Brace, nor seen as yet from the islands.) 
Phyllanthus bahamensis Urb.

In coppices :

Abaco - California Road, Brace 2037.

Andros - at Red Bays, Northrop 488.

New Providence - near Nassau, Curtiss 183; Waterloo, B. @ Br. 741 ;

E. G. B. 3435 ; Farringdon Road, E. G. B. 3403.

Eleuthera - Coker 328.

\section{Phyllanthus distichus L.}

Escaped from cultivation:

Andros - Fresh Creek, Northrop 653, where it is known as "GooseBERRY TREE."

\section{Phyllanthus epiphyllanthus L.}

$(P$. falcatus $S z i$.) In various situations throughout the islands from Andros southward:

Andros - Morgan's Bluff, where it is known as "Hardhead," Northrop $146 a$.

North Bimini-Millsp. 2375 .

South Bimini-Millsp. 2411.

South Cat Cay - Millsp. 2426.

Silver Cay - Millsp. 2453.

New Providence - B. o Br. 223, 520; Britton 14, 57; Coker 31; Northrop 325 ; Cooper 48; Earle 57 ; Curtiss 4; Brace 58, 230 ; Hitchcock; Millsp. 2068, 2081, 2132; Wight 18.

Eleuthera-Governor's Harbor and Palmetto Sound, Hitchcock.

Cave Cay- $B$. \& $M$.

Little Galiot Cay - B. \& M. 2837.

Great Guana Cay-B. \& M. 2866, 2919.

Exuma - B. \& M. 3034, 3080 .

Cat Island - Hitchcock.

Fortune Island - Hitchcock.

Water Cay - Coker 524 .

Watlings Island - Hitchcock.

Inagua $-N$. \& T.875; Hitchcock.

Grand Turk Island $-N$. \& $T .3766$.

Varies from very long, slender leaves, through broader forms to short, broad, neariy ovate phyllodes.

\section{Phyllanthus niruri L.}

Open sandy places general :

Abaco - Cherokee Sound, Brace 2083.

Little Harbor Cay - B. ㅇ M. 2252. 
Great Harbor Cay - B. \& M. 2338.

Andros - Nichols Town, Northrop 338; Wight 265.

North Bimini - Millsp. 2400.

New Providence - E. G. B. 3296 known as "Gale of Wind"; $B$. \& Br.

736; Curtiss 68; Brace 260; Wight 93.

Mangrove Cay - Coker 227.

Great Guana Cay $-B$. o $M$.

Exuma $-B$. \& $M$.

Fortune Island - Hitchcock.

Inagua $-N$. ڤ T. 1376; Hitchcock.

Grand Turk Island $-N$. \& T. 3866 .

Phyllanthus radicans (Muell Arg.) Small.

In pine barrens :

Great Bahama - Eight Mile Rocks, B. \& M. 2381 .

Phyllanthus saxicola Small.

On coral rock or sands in open places. The type is from South Florida between Coconut Grove and Cutler, Small \& Carter 775 in herb. N. Y. Bot. Garden.

Great Bahama - Eight Mile Rocks and Barnett's Point, B. \& M. 2489, 2623 (simple stemmed).

Great Sturrup Cay - B. \& $M .2273$ (simple stemmed).

Little Harbor Cay - B. \& M. 2250.

Great Harbor Cay - B. \& $M .2333$.

New Providence - at Tea House, E. G. B. 3447; near Nassau, Curtiss 79. Great Guana Cay - B. \& M. 2861 .

\section{Drypetes sp.}

Specimen in leaf only :

Exuma - in a rocky coppice near Georgetown, B. \& M. 2984. Apparently quite a different species from the following two. Leaves ovate narrowing to a prolonged sharp point, reticulate on both surfaces, upper surface dark, shining, lower surface pale, smooth. 4-6.x 2-I.5 $\mathrm{cm}$., petioles about $.5 \mathrm{~cm}$.

Drypetes diversifolia Kr. \& Urb.

D. keyensis Kr. \& Urb. See remarks upon this species by Dr. Britton in Bull. N. Y. Bot. Gard. 3: 444, since which the following new localities have been determined:

Great Bahama - West End, Brace 3618.

Lignum Vitae Cay - B. \& M. 2319.

Little Harbor Cay - B. \& M., where it is known as "White Wood."

Whale Cay $-B$. \& $M$. 
New Providence - at Winton, E. G. B. 3290.

Wide Opening, Cay north of $-B$. \& $M .2760$.

Little Galiot Cay - $B$. \& $M$.

Stocking Island $-B$. \& $M$. 3060 .

Exuma - $B$. \& $M$.

Drypetes laterifiora (Sw.) Kr. \& Urb.

Maritime sandy coppices:

Elbow Cay - Brace 1688.

Great Harbor Cay - B. \& M. 2350.

New Providence-coppice on the north side of Blue Hills, E. G. $B$. 3452., the Race Course 3421, and at Winton 3293; Eggers 4331.

Croton cascarilla (L). Linn.

This species, first depicted by Catesby vol. 2 plate 46 , has only been collected once since, then on New Providence by Mr. W. F. Daniell, whose specimen, as seen by Dr. Britton, proves Catesby's drawing to be a good representation of the species. All other reports of the species from the Bahamas or Antillean Islands are in error and are principally based upon forms of Croton linearis. Prof. Hitchcock's specimens are not this species.

\section{Croton discolor Willd.}

So far known from only one station:

Rum Cay - Coker 453 at Port Nelson.

Croton eluteria (L) Sw.

In coppices and scrub lands, where it is known as "CASCARILlA" or "SweEtwood Bark." This is the $C$. niveus of Gardner \& Brace:

Great Harbor Cay $-B$. \& $M$. 2339.*

Hog Island-Eggers 4151 .

New Providence-Daniell; Brace 56, 289; Curtiss 185; Coker 3a; E. G. B. 3143 .

Eleuthera - Eggers 4425; Hitchcock, at Governor's Harbor.

Exuma - rocky coppices near Georgetown, B. \& $M .2985$.

\section{Croton flocculosus Geis.}

So far known from only one island:

New Providence - near Nassau, Curtiss 61; Brace 516.

*Dr. Britton finds that Wright's type of Croton homolepidus in herb. British Museum is the same as B. \& M. 2339, and identical with Catesby's specimen of C. eluteria. 
Croton Hjalmarssoni Griseb.

In sandy opens and whitelands :

Great Guana Cay - B. \& M. 2891.

Fortune Island - Hitchcock.

Inagua $-N$. \& T. 874,882 ("compares with the type in Herb. Kew"Britton); Hitchcock.

Grand Turk Island $-N$ : \& $T .3782,3802$.

\section{Croton humilis Linn.}

The only specimens that we have seen from the Bahamas are:

Cat Island - at Port Howe, Hitchcock.

Croton linearis Jacq.

Maritime sand plains and dunes from the Berry Islands southward, where it is known generally as "Granny Bush":

Great Sturrup Cay $-B$. \& $M$.

Little Harbor Cay - B. \& M. 2244.

Great Harbor Cay $-B$. \& $M$.

Whale Cay-B. \& $M .2184$.

South Bimini - Millsp. 2407.

Gun Cay - Millsp. 2315.

Andros - Fresh Creek, Northrop 615.

Rose Island $-B$. \& $M .21 / 3$.

New Providence - Northrop 113; Cooper sine num.; Coker 23; B. \& Br. 304; Britton 85; Millsp. 2154; Wight 65.

Eleuthera - Governor's Harbor, Hitchcock. (C. cascarilla linearis); Coker 387.

Great Guana Cay $-B$. \& $M$.

Little Galiot Cay - where it occupies the southwest plateau almost to the exclusion of all other vegetation, $B$. \& $M .2844$.

Exuma - in scrublands north of Georgetown, B. \& M. 2942.

Cat Island - at Port Howe, Hitchcock.

Watlings Island - Hitchcock (C. cascarilla L.). This specimen is a counterpart of a sheet of C. linearis from Palı Beach, Florida, Curtiss 5360, in Herb. Field Col. Mus.

Inagua $-N$. \& $T$. 906 , these specimens have very long and narrow leaves, and smaller seeds than the usual form.

Croton lobatus Linn.

In cultivated soils. This species can be unhesitatingly placed among the Antillean weeds that have been more or less lately introduced in the Bahamas :

New Providence-Earle 32; B. \& Br. 783; Coker 553; Brace 353. 


\section{Croton lucidus Linn.}

Sandy scrub and white-lands near the sea :

Great Sturrup Cay - B. \& M. 2283.

Great Harbor Cay - B. \& $M$.

New Providence - near Clifton, E. G. B. 3323; Cooper 22; Britton 21, 82; Brace 68, 288, 3896; Curtiss 108; Millsp. 2077, 2156; Wight 109, 173.

Eleuthera - Coker 326.

Exuma - B. \& M. 2930.

Watlings - Coker 471 .

Croton rosmarinifolius Griseb.

One locality only is known up to this date :

Exuma - abundant in one situation, an open, rocky field just north of Georgetown, where it forms small, bushy trees up to $3.5 \mathrm{~m}$. high, B. \& M. 2978 .

Our specimens compare exactly with Wright's, Cuba, 1968 in Hb. Kew.

\section{Argythamnia argentea sp. nov.}

Fruticulus ad basi ramosissimus, rami virgatis subdense foliosis, ramuli canescentibus. Foliis lanceolatis utraque attenuatis breve petiolatis margine superne pauco-dentatis utraque pagina argenteosericeis; stipulis crassis cuspidatis. Racemulis depauperatis supra axillaribus. Flores masc. 3, sepalis 4 ovato-lanceolatis, petalis 4 crassis, sepalis similiter sed brevissimis, bracteola I scaphoideis; flores foem. 3 , sepalis elongato-lanceolatis, petalis minute ligulatis glandulae alternantibus; stylis 3 , prox basi bifurcatis ad apice bilobatis, ovario canescentibus.

Near A. sericea from which, however, its characters plainly separate it. The type and only plants so far seen are from:

Grand Turk Island-in scrubland Aug. 27-Sept. I, 1905, Nash \& Taylor 3830 .

\section{Argythamnia candicans Sw.}

So far only known in the Bahamas through the following specimens that are clearly this species :

Cat Island - Port Howe, Hitchcock.

Inagua - Hitchcock.

\section{Argythamnia lucayana sp. nov.}

Fruticulus ramosis prostratis vel ascendentibus. Rami minute sericeis sparse foliosis, foliis sessilis elliptico- velobovato-lanceolatis apice acute vel brevissime acuminatis margine integris vel praeter admodum paucos minute et remote glanduloso-denticulatis, matura glabris imma- 
tura subtus sparse et minute strigose pilosis. Racemuli $4^{-6}$ floris. Sepalis masc. deltoideo-lanceolatis petalem aequantibus, petalis spathulatis, pilosis, staminis 4, laevis, calycis foem. laciniis foliaceis lanceolatis acuminatis, petalis minutis, angustis-lanceolatis obtusis paleaceis calyce pluries brevioribus, ovario villoso-pubescentis, stylis villosis bis dichotome divisis, dichotomiis terminiis brevis. Semen ad maturitas globosis, apiculatis, anastomoso-reticulatis.

A small shrubby plant with branches $3-5 \mathrm{dm}$. long. Leaves bluishgreen $2.5-3.5 \mathrm{~cm}$. long, $\mathrm{r}-\mathrm{I} .5 \mathrm{~cm}$. broad. Racemes 6 - ro $\mathrm{mm}$. long, 4-6 flowered. Seeds I.5 mm. in both diameters, dark brown, the surface coated with a hydnum-like porous integument.

This is the common Argythamnia of the Bahamian islands, where it is to be found on the floor of the coppices or at times in more open situations:

Great Sturrup Cay - B. \& M. 2280.

Little Harbor Cay - B. \& M. 2232.

Eleuthera - Tarpum Bay, Coker 409 (A. candicans).

Ship Channel Cay - B. \& $M .2760$.

Wide Opening, Cay north of $-B$. \& $M .2789$.

Cave Cay - B. \& M. 2834.

Great Guana Cay - in rocky coppice, $B$. \& $M .2913$ type.

Little Galiot Cay - B. \& M. 2839.

Exuma - B. \& $M .3601$.

\section{Argythamnia sericea Griseb.}

'The only island upon which this species has as yet been found in typical form is :

Inagua - in sand on whitelands at Tenados, Oct. I4, I904, N. \& T. I030.

A striking form with flabellate leaves and minor character differences is returned from:

Fortune Island-Brace 462.

\section{Mercurialis annua L.}

Cultivated ground:

New Providence - Wight II 8 .

This is the first return of this species from the Bahamas.

Bernardia bernardia (L.) Britton.

Coker in Shattuck's "Bahama Islands" p. 257. Adelia Bernardia L. Bernardia carpinifolia Griseb., Bernardia dichotoma Mill.

Eleuthera - at Tarpum Bay, Coker 405; Governor's Harbor, Hitchcock (B. mexicana).

Cat Island - Port Howe, Hitchcock (B. mexicana).

Fortune Island - Brace 458 . 


\section{Lasiocroton macrophyllus Griseb.}

So far known only from Mrs. Northrop's specimens which, while not absolutely agreeing with Marsh's Jamaican specimen in Hb. N. Y. Bot. Garden, are undoubtedly the species. The sheet in Hb. Field Col. Mus. is in good flowering condition.

Andros - at Deep Creek, Northrop 689, where it is known as "WILD OAK," "Lightwood" or "Bitters".

\section{Acalypha alopecuroidea Jacq.}

Doubtless an introduced weed in waste and cultivated grounds:

Abaco - at Marsh Harbor, Brace 1751.

New Providence - near Nassau, Northrop 32; Curtiss 22; Hitchcock;

Wight 39; Brace 132, and at Southwest Landing, B. \& Br. 474.

Eleuthera - at Governor's Harbor, Hitchcock.

Inagua - at Mathew Town, $N$. \& T. 1080.

Acalypha ostryaefolia Ridd.

Known only by the following plant, probably introduced from Florida:

New Providence - at Nassau, Brace 385 (300?)

Acalypha setosa A. Rich:

Known only by the following plant, doubtless an introduced weed.

New Providence - at Nassau, Brace 299 (267?).

Pera bumeliaefolia Griseb.

In coppices; known as "Black ЕвоNY":

Abaco - at Marsh Harbor, Brace 1603.

Andros-Brace $4 I I$.

New Providence-coppice at Seven Hills, E. G. B. 3360 the forma parvifolia of Urban in Hb. Kew.; Fox Hills, B. \&o Br. 376; near Nassau, B. \& Br. 267; Coker 537; Curtiss sine num., Brace 226; Wight 166.

\section{Ricinus communis Linn.}

Escaped from gardens on:

New Providence - Wight 160 .

Fortune Island - Hitchcock.

\section{Jatropha curcas Linn.}

Known only by the following specimen, probably an escape from a garden :

New Providence - near Nassau, Brace 305.' 
Jatropha gossypiifolia Linn.

The only specimens seen are from waste lands near Nassau:

New Providence - Hitchcock; Brace.

Manihot manihot (Linn.) Cockl.

Manihot apii Pohl. Known only as cultivated at :

Andros - Nichols Town, Northrop 363 .

Eleuthera - Governor's Harbor, Hitchcock.

Excoecaria Sagraei Muell.

In coppices. The plant has a strong mephitic odor:

Andros - Stafford Creek, Northrop 539.

New Providence-Curtiss 190; $B$ \& Br. 665 ; Fox Hills Road, E. G. B. 3388; Britton 78; Millsp. $2160,2486$.

Exuma - B. \& $M .3079$.

Watlings Island-Coker 478 .

Bonania cubana A. Rich.

In coppices and scrub lands:

Andros - at Fresh Creek, Northrop 628 (B. emarginata); Wight 254.

New Providence - in a coppice at Winton, E. G. B.3294; Brace 141, 340.

Cat Island - in a low, dry coppice at Arthur's Town, Coker 428.

Exuma - near Rolletown, B. \& $M .3074$.

Hippomane mancinella Linn.

In coppices and scrub lands. Known generally as "MANCHineEL" or "Poison Bush."

Abaco - Cherokee Sound, Brace 1920.

Andros - Fresh Creek and Conch Sound, Northrop 622, 565 (misprinted 556 in Fl. N. P. \& Andros).

Great Guana Cay $-B$. \& M. 2873.

Watlings Island - Coker 485 .

Inagua - at Lantern Head and Tenados, N. \& T. 1392, 1048; Hitchcock.

Grand Turk Island - in scrublands, $N$. \& $T .3826$.

Gymnanthes lucida Sw.

Coppices and scrublands. Known generally as "CRABwóoD":

Allen's Cay - Brace 1547:

Green Turtle Cay - Brace 1512.\$

Great Bahama - at Barnett's Point, B. \& M. $2642 . \ddagger$

Great Harbor Cay $-B$. \& $M$.

Lignum Vitae Cay $-B$. \& $M$.

Great Sturrup Cay $-B$. \& $M$. 
Goat Cay $-B$. \& $M$., where it forms character thickets.

Whale Cay $-B$. \& $M$.

South Bimini - Brace 3476.

Gun Cay - Millsp. $2327 .^{\circ}$

Cat Cay - Brace $3751 . \dagger$

Andros - at Fresh Creek and Nichols Town, Northrop $375 ;^{\circ}$ Wight $248 .^{\circ}$

Rose Island $-B$. \& $M$.

New Providence - near Clifton, E. G. B. 3326; coppice at Lake

Cunningham, B. \& Br. 606, $607 ; \S$ Fox Hills, B. \& Br. $862 ;^{\circ}$ E. G. B. $3393 ;^{\circ}$ Britton $103 ; \S$ Brace 299,† $335 ; \dagger$ Curtiss 190; Millsp. $2183 . \S$

George's Island - Spanish Wells, Coker 314. $\dagger$

Ship Channel Cay - B. \& $M$.

Wide Opening, Cay north of $-B$. \& M. 2804 $\ddagger$

Great Guana Cay $-B$. \& $M$.

Exuma $-B$. \& $M$.

Watlings Island - Coker $467 . \dagger$

Inagua - on Salt Pond Hill, N. \& T. 927,* 992.*

Sheep Cay - N. \& T. 1145.*

Hura crepitans Linn.

Cultivated, only at Nassau.

\section{Euphorbia Berteriana Balbis.}

So far seen only in the redlands of:

Exuma - B. \& M. 2938, 3031 .

Euphorbia Blodgettii Engelm.

On rocks, sand dunes, whitelands and in pot holes common. Not yet found on the islands of the Abaco group.

Great Bahama - B. \& M. 2383, 2470, 2619.

Great Sturrup Cay-B. \& M. 2257, 2272, 2274.

Frozen Cay $-B$. \& $M .2216$.

Whale Cay - B. \& M. 2182.

North Bimini - Millsp. 2404; Brace 3462.

South Bimini-- Millsp. 2364 .

Gun Cay - Millsp. 2310.

Andros - Nichols Town, Northrop 379.

Salt Cay - Northrop 277 (E. serpens).

Hog Island - B. \& $M .2169,2174$.

Rose Island $-B$. \& $M$. 2146 . 
New Providence - Cooper sine num.; Millsp. 2100, 2149, 2150, 2167, 2271 ; Curtiss 70 (E. Nashii Small); Britton 72, 74, 90; B. \& Br. 195, 279, 309, 313, 340, 400, 477, Northrop 41; Hitchcock.

Eleuthera - Governor's Harbor, Hitchcock (3 sheets).

Ship Channel Cay - B. \& $M$. 2746, 2755,

Great Guana Cay - B. \& $M .2863$,

Cave Cay - B. \& $M .2805,2818$.

Great Galiot - B. \& $M .2859$.

Exuma-B. \& $M$. 2961, 2966, 3107, 3112.

Fortune Island - Hitchcock.

Crooked Island - Hitchcock.

Inagua $-N$. \& $T$. 954, 1090, 1435; Hitchcock.

Sheep Cay $-N$. \& T. 1159 .

Grand Turk Island - N. \& T. 3851,3860 .

\section{Euphorbia Bracei sp. nov;}

$\S$ Chamaesyceae. Caulibus prostratis ad basin ramosis, rami lignosis canescentis, foliis petiolatis oppositis oblongis vel ovatis ad basin oblique-deltoideis apice rotundo-obtusis margine integris revolutis utrinque pagina hirsutis et resinosis, stipulis triangulo-lanceolatis acutis, involucris axillaribus, cupuliformis, pedicelis tubo aequantibus, extus canescentis intus ciliatis, mure crassis interiore in columnae alaribusque 5 productis, lobis triangularis acutis, glandulis minutis transverseovatis appendice angustissima vel obsoleta crenato- 3 dentatis, stigmatis ad basin bifurcatis. Capsulae aciculo-hirsutis, semen trianguloovoideis leviter anastomo-rugosis.

A low, grayish plant near E. cayensis Millsp. from which it differs in many characters. Leaves $8 \times 5$ to $5 \times 3 \mathrm{~mm}$. dotted with minute amberlike resin globules; petiole one-fifth the length of the blade; internodes I.3-2 cm.; fifth gland replaced by a broad fifth tooth equalling the breadth of a gland; seed $.9 \times .7 \mathrm{~mm}$., the angles prominent.

Abaco - in sand at Eight Mile Bay, Brace 1856 type.

Man o'War Cay - on whitelands, Brace 1571 .

\section{Euphorbia brasiliensis Lam.}

In open places and grassy coverts:

New Providence - at Lake Cunningham, Britton 137; Millsp. 2227; cultivated ground, Farringdon Road, E. G. B. 3199.

Eleuthera-at Gregory Town, Coker 371 .

\section{Euphorbia Brittonii sp. nov.}

$\S$ Chamaesyceae. Pilosa praegracilis erectis ad basin ramosis, rami filosis ascendentibus, foliis oppositis breviter petiolatis rotundatis vel oblongo-ellipticis apice rotundatis basi leviter et oblique cordatis margine integerrimis vel acute subdentatis revolutis obsolete venosis nervo 
medio subtus prominentis, crassiusculis, pagina subtus pilosis glaucoviridis; stipulis triangulatis plus minusve aequaliter fimbriatis; involucris solitariis axillaribus, campanulatis, pedicellatis, extus glabris intus ad faucam dense tomentosis, lobis triangularis inflexis praeciliatis, glandulis ovatis albo-viridis, appendice magnis orbiculatis albis. Stamina insertis pilosis (paleis linearibus glabris intermixtis), stigmatis supra bifurcatis; capsulae glabris; semina roseo-cinereis ovatis valide triangulatis leviter transverse rugosis.

A small, erect, wiry plant branching from the base, $5-7 \mathrm{~cm}$. high; leaves $4 \times 3 \mathrm{~mm}$. glands 4 the fifth replaced by a sharp fissure flanked by 2 larger involucral lobes.

So far known only from sandy whitelands, as follows:

New Providence- - at the Race Course, Aug. 26, r904, B. \& Br. 839 type and 279.

\section{Euphorbia buxifolia-I_am.}

Maritime beaches from Great Bahana southward.

Green Turtle Cay - Brace 1508.

Abaco - Cherokee Sound, Brace 1902, and Butler Bay, 1520.

Great Bahama - infrequent, low and undeveloped. Eight Mile Rocks, in a clearing near the sea, $B$. \& $M .2781$.

Great Sturrup Cay - B. \& M. 2256.

Great Harbor Cay $-B$. \& $M$.

Lignum Vitae Cay - B. \& $M$.

Frozen Cay $-B$. \& $M$.

Whale Cay $-B$. \&o $M$.

Gun Cay - Millsp. 2317.

South Cat Cay - Millsp. 24I7, 2418 .

Andros - at Red Bays and Big Cabbage Creek, Northrop 457, 672.

Silver Cay - Millsp. 2454.

Rose Island $-B$. \& $M$.

New Providence - South shore, Millsp. 2109; near Nassau, Cooper sine num.; E. G. B. 3185; Northrop 87; Coker 114; Hitchcock; Wight 55. Eleuthera - Governor's Harbor, Hitchcock.

Ship Channel Cay - B. \& $M$.

Wide Opening, Cay north of $-B$. \& $M .2781$.

Great Guana Cay $-B$. \& $M$.

Great Galiot Cay $-B$. \& $M$.

Little Galiot Cay $-B$. \& $M$.

Cave Cay - B. \& $M$.

Exuma - B. \& $M$.

Stocking Island $-B$. \& $M$.

Cat Island - Port Howe, Hitchcock. 
Watlings Island - Hitchcock.

Crooked Island - Hitchcock.

Fortune Island - Hitchcock.

Inagua $-N$. \& $T \cdot 1038$.

Sheep Cay $-N$. \& T. i i 60 .

Grand Turk Island $-N$. \& $\dot{T} \cdot 3793,3889$.

Euphorbia cassythoides Boiss.

Known so far only from the following specimens:

Andros - Deep Creek, Northrop 702.

\section{Euphorbia cayensis Millsp.}

Maritime sands. So far found only on the smaller cays from Joulter's Cays northward.

Man o'War Cay - Brace 1571.

Great Sturrup Cay - B. \& M. 2266.

Great Harbor Cay - B. \& $M .2334$ (prostrate).

Little Harbor Cay $-B$. \& $M$.

Frozen Cay $-B$. \& $M$.

Whale Cay $-B . \& M .2178$, where it is the character plant of the maritime whitelands, and is known as "Milx Bush."

Joulter's Cays - Millsp. 2295 type.

\section{Euphorbia flexuosa $\mathrm{K}$ th.}

E. buxifolia flexuosa (Kth.) Boiss. Anyone seeing this species in the field would at once recognize it as clearly distinct from E. buxifolia. Its purplish coloration, cinereous glaucescence, rounded flat leaves and spreading habit cause it to resemble E. Cayensis far more closely than E. buxifolia.

Leaves glauco-cinereous, ovate, rounded at the apex and unequal base $.3-1 \mathrm{~cm} . \times \cdot 2-.6 \mathrm{~cm}$. Involucres turbinate, short pediceled, smooth without, densely bearded within, margin entire; glands 4 , large, cordate, nearly equalling the appendages; stamens 5 , the filaments bearded and tufted below; seeds triangular, pink-ashen, farinose, the angles rounded and prominent, the facets slightly anastomose-rugose transversely.

Maritime sands and sand-pocketed rocks. Not seen north of the Exuma Chain.

Ship Channel Cay - B. \& M. 2747.

Wide Opening, Cay north of $-B$. \& $M .2777,2779$, On the open seaside of this cay the plants are strongly characteristic and to be found in great quantity both erect and prostrate.

Little Galiot Cay $-B$. \& $M$. 
Stocking Island $-B$. \& $M .3071$.

Rum Cay - Coker 452 (E. cayensis). M̀r. Coker mentions seeing this plant also on Abaco, but doubtless mistook the Abacan E. Bracei for this species.

\section{Euphorbia havanensis Willd.}

E. heterophylla graminifolia Eng. This species as it occurs in South Florida and the Keys, Cuba and the Bahamas, is plainly distinct from E. heterophylla.

Green Turtle Cay - Brace 1479.

Great Sturrup Cay - B. \& M. 2255.

New Providence - Northrop 92; Millsp. 2146; Britton 69; Coker 45; Wight 14.

Eleuthera - Governor's Harbor, Hitchcock (E. heterophylla graminifolia). Exuma - near Georgetown, B. \& $M .3133$.

Cat Island - Port Howe, Hitchcock (E. heterophylla graminifolia).

Euphorbia heterophylla Linn.

This species appears to exist in all its forms in the Bahama Islands where it is principally found in open waste grounds.

Green Turtle Cay - Brace 1503, 1479.

Great Bahama - Eight Mile Rocks in pine barren, $B$. \& $M .2461$; West End, Brace 3499.

Little Harbor Cay - B. \& $M$.

South Bimini - Millsp. 2365.

South Cat Cay - Millsp. 2435.

New Providence - near Nassau, Northrop 96; Millsp. 2204; Hitchcock; Wight 29.

Eleuthera - Governor's Harbor, Hitchock.

Great Guana Cay - B. \& $M$.

Little Guana Cay $-B$. \& $M$.

Exuma - in a cornfield, $B$. \& $M$.

The form with the lower (and often all) leaves broadly ovate-lanceolate, entire or obsoletely repand, E. prunifolia Jacq. This latter form plainly belongs to E. heterophylla rather than to E. geniculata Ortega. Under this form the following specimens may be grouped:

Green Turtle Cay - Brace 1539.

South Cat Cay - Millsp. 2426.

New Providence - near Nassau, Earle 33; Curtiss 73; Hitchcock (E. geniculata); Wight 5 .

Eleuthera - Governor's Harbor, Hitchcock (E. geniculata).

Cat Island - Port Howe, Hitchcock (E. geniculata).

Fortune Island - Hitchcock (E. geniculata). 
Inagua - Mathew Town, $N$. œ T. 1086; Hitchcock (E. geniculata).

Abaco - Eight Mile Bay, Brace i 870.

Euphorbia hypericifolia Linn.

In grassy open lands :

Great Bahama - Eight Mile Rocks, B. \& M. 2476.

North Bimini - Millsp. 2370, 2390.

Andros - Nichols Town, Northrop 380 (E. nutans).

New Providence - at Lake Cunningham, Millsp. 2227; near Nassau, Earle $46^{\mathrm{a}} ; \mathrm{B}$. \& Br. 470, 535; Hitchcock; Wight 46.

Eleuthera - Governor's Harbor, Hitchcock.

Great Guana Cay - B. \& M. 2906.

Exuma - near Georgetown, B. Ev $M .2945$.

Inagua - Smith's Thatch Pond, N. \& T. 1401, 1406; Hitchcock.

Euphorbia lactea Haw.

Widely escaped from cultivation and forming dense thickets on:

New Providence-along thẹ West Bay Street Road, Northrop (E. antiquorum).

Inagua - Mathew Town, Hitchcock (E. antiquorum).

\section{Euphorbia lecheoides sp. nov.}

Tota griseo furfureo-farinoso caulibus e rhizomate lignoso, ramosissimo. Rami longis, diffusis rigidis dichotomis, internodiis inferioribus prox. I cm. longis, superne tenuiter virgatis rigidis. Foliis breviter petiolatis, deltoideo-ovatis vel triangulari-ovatis abrupte attenuatis ad margine revoluto-intumescentis, pagina utruque furfureo-farinosis, stipulis late ovatis blepharociliatis, involucris solitariis turbinatis brevi pedicellatis, extus glabris intus ad faucam barbatis, lobis triangularibus acutis ciliatis, glandulis nigris complano-orbiculatis, facie notis variolarum insignis, appendices minutis carnosis vel obsoletis, stigmata ad mediam bifurcatis, filamentae barbatis. Capsulae glabris. Semen fuscis triangulo-ovatis fascies minute papillatis.

Plants rigidly erect or diffusely prostrate $20-30 \mathrm{~cm}$. Leaves 2.5 to $5 \mathrm{~mm}$. long, I to $2.5 \mathrm{~mm}$. wide. Seeds .8 $.5 \mathrm{~mm}$. The specimens representing the extremes of this species are so widely different in general appearance as to almost convince one that they represent two strongly marked species. This difference, however, lies entirely in the habit of the plants and the size of the leaves. The tallest erect form with small, ovate leaves $\left(379^{6}\right)$ passes through the type (3888) to a more diffuse, larger leaved form (I 305 ) and a fully prostrate plant with much larger, sickle-shaped leaves ( $1 \mathrm{~cm}$. $\times 3 \mathrm{~mm}$.) with a somewhat less intumescent margin.

Inagua - in scrubland on the trail from Canfield Bay to Cabbage Pond, Oct. 22, I904, Nash \& Taylor 1305. 
Grand Turk Island - in sandy scrublands, Nash \& Taylor 3888 type and on the strand, 3796 .

\section{Euphorbia obliterata Jacq.}

This species, considered a synonym of E. pilulifera procumbens by Boissier, is distinct both in characters and in habitat.

Great Bahama - at Eight Mile Rocks, B. \& M. $247 I$.

Little Harbor Cay - B. \& M. 2247.

New Providence - on coral limestone (the usual habitat) along Farringdon Road, B. \& Br: 199, and Soldiers Road 807; Wight 179.

Cave Cay - B. \& $M .2817$.

Exuma - B. \& $M .2938,3031$.

Inagua - Hitchcock (E. pilulifera obliterata.)

Euphorbia pilulifera Linn.

Frequent in cultivated grounds and along roads and paths:

New Providence - B. \& Br. 757, 805; Millsp. 2216; Hitchcock.

Eleuthera - Governor's Harbor, Hitchcock. .

Cat Island - Port Howe, Hitchcock.

The prostrate form with the same characteristic jointed amber colored hairs (E. pilulifera procumberis Boiss.) from:

New Providence $-B$. \&. Br. 757.

Inagua $-N$. \& $T$. 1088 .

\section{Euphorbia prostrata Aiton.}

So far known only from two localities:

New Providence - near Nassau, Curtiss sine num.

Grand Turk Island $-N$. \& $T .3871$.

Euphorbia punicea Sw.

On the southern islands only:

Watlings Island - on a small cay in the lake, Coker 487.

Fortune Island - Hitchcock.

Inagua $-N$. \& $T$. $1263,1446$.

Little Inagua $-N$. \& $T$. 1216 .

Euphorbia trichotoma Lam.

This species, common on the maritime sands of the Florida Keys and the Antillean Islands, is so far only known in the Bahamas by specimens collected on:

Allen's Cay - Brace 1551.

Great Bahama - at Eight Mile Rocks, B. \& M. 2364; West End, Brace 3493 . 


\section{Euphörbia vaginulata Griseb.}

The following specimens represent the important rediscovery of this endemic species.

Little Inagua - Moujean Harbor, $N$. \& T. 1222.

Inagua $-N$. \& $T$. 1170,1304 .

Grand Turk Island - Hjalmarsson (type,) N. \& T.3764.

The plants grow in great profusion on Grand Turk Island. The Inagua specimens, while apparently differing from those of Grand Turk Island and Little Inagua on account of their longer leaves (twice or thrice the length) and darker bark, have the same characters as the type.

\section{Pedilanthus tithymaloides (L) Poit. ?}

In coppices, rare:

Andros - at Deep Creek, Northrop 693 (P. angustifolius?)

Inagua - at Mathew Town, $N$. \& $T \cdot 1363$.

Grand Turk Island $-N$. \& $T: 3761$.

Plants from Grand 'Turk Island are now growing in the conservatory of the Garden which give promise of settling upon the specific character of this form.

\section{RUBIACE E.}

Rachicallis maritima (Jacq.) K. Sch.

R. rupestris (Sw.) D. C. On maritime rocks throughout the Islands. Often the only vegetation on many of the sea washed islets. Observed by $B$. \& $M$. and Millsp. on each island visited. The collections contain the following sheets:

Spanish Cay - Brace 1562.

Great Bahama - Allen 36.

South Cat Cay - Millsp. 2425.

North Cat Cay - Millsp. 2415.

Andros - Red Bays and Fresh Creek, Northrop 458 (R. Americana) where it is known as "Sand-fly Bush," "Salt Water Bush," "Seaweed" and "Wild Thyme."

New Providence-Cooper 2; B. \& Br. 293; Curtiss 32; Coker 19; Wight 190; Millsp. 2249; Brace 161, 165, 173; Hitchcock (R. Americana.)

Crooked Island - Hitchcock (R. Americana).

Inagua - $N$. \& $T .904,1366,1391$.

Grand Turk Island. $-N$. \& T. 3812 .

Exostema caribaeum (Jacq.) Willd.

In shady coppices. Known as "Prince WoOD.

Elbow Cay - Brace 1698. 
Abaco - Cherokee Sound, Brace 1930.

Great Bahama - Eight Mile Rocks and Golden Grove, B. \& M. 2433, 2733; West End, Brace 3516.

Andros - Kemp Sound and Deep Creek. Northrop, 685.

Rose Island $-B$. \& $M .2117$.

New Providence - Fort Montague, $B$. \& Br. 316; Brace 187; Fox Hills, E. G. B.'3392.

Wide Opening, Cay north of $-B$. \& $M .2787$.

Exuma $-B$. \& $M$.

Watlings Island - Coker 465 .

Crooked Island - Hitchcock.

Inagua $-N$. \& $T$. 952, 1431 .

Genipa clusiaefolia (Jacq.) Griseb.

Coastal rocks of all islands visited $(B$. \& M., Millsp.). Known generally as "Seven Year APple."

Andros - Mastic Point, Northrop 299.

North Cat Cay - Millsp. 2346.

New Providence - Brace 41; Millsp. 2113; Hitchcock.

Watlings Island $\rightarrow$ Hitchcock.

Cat Island - Hitchcock.

Crooked Island - Hitchcock.

Fortune Island - Hitchcock.

Inagua $-N$. \& $T .937,1345$.

Little Inagua $-N$. \& $T$. 1183 .

Randia aculeata Linn.

In thickets, coppices and scrublands. The farther south in the Archipelago the plants are found the smaller the leaves appear. The spinous or aspinous character is not, however, so constant. Not seen on the Bimini Group.

Elbow Cay - Brace 1703.

Abaco - Cherokee Sound, Brace 1918.

Great Bahama - Eight Mile Rocks, B. \& M. 2432, West End, Brace 3540. Berry Islands - on all islands visited except Frozen and Goat Cays, $B$. \& $M$.

Little Harbor Cay - B. \& M. 2225.

Cat Cay - Brace 3743 .

Andros - Nichols Town, Northrop 383; Wight 252.

Rose Island $-B$. \& $M$.

New Providence-B. o Br. 323; Coker 56; Hitchcock.

Exuma Chain - noted on all islands visited except Great Guana Cay, B. \& $M$. 
Eleuthera - Palmetto Sound, Hitchcock.

Cat Island - Port Howe, Hitchcock.

Crooked Island - Hitchcock.

Inagua $-N$. \& $T$. 1060,1062 with very small leaves; Hitchcock.*

Grand Turk Island $-N$. \& T. 3759 .

Hamelia patens Jacq.

Waste grounds near habitations. This habitat, and the absence of the species from other islands, place it as a doubtless escape from cultivation.

New Providence - Lake Cunningham, B. \& Br. 635; Bluebeard's Tower, Coker 127; Nassau, Northrop 40; Wight 147.

Catesbaea campanulata La Sagra.

C. parvilora septentrionalis Urb. In coppices and scrub lands:

New Providence - Brace 167.

Crooked Island - Hitchcock (C. parviflora). See remarks on Grand Turk Specimen below.

Inagua $-N$. \& $T$. 1272 .

Grand Turk Island $-N$. \& $T .3771$. This is a low, scrubby specimen having no spines developed upon the short new shoots. It appears to be a depauperate mutant with narrower and more revolute leaves and smaller seeds than the typical form.

Catesbaea fasciculata Northrop.

In sandy coppices and whitelands:

Abaco - Eight Mile Bay, Brace 1852.

Little Harbor Cay - B. \& M. 2226.

Great Harbor Cay - B. \& $M$.

Andros - Fresh Creek, Northrop 627 type.

New Providence - scrublands along West Bay Street Road, Britton 83; Millsp. 2162; coppice at Winton, E. G. B. 3292; whitelands near Race Course and Miller's, $B$. \& $B r .284,529$.

Green Cay - maritime beach, Coker 247.

Ship Channel Cay - B. \& M. 2750.

Wide Opening, Cay north of $-B$. \& $M$.

Great Guana Cay - B. \& $M$.

Catesbaea spinosa Linn.

Sandy open lands:

Andros - at Fresh Creek, Northrop 624. Known as "Prickly Apple."

*Prof. Hitchcock observes on p $93 \mathrm{Pl}$. Bah.: "The Inagua specimens are spineless and have small leaves about $7 \mathrm{~mm}$. long." The Nash \& Taylor specimens have such leaves and also spines. 
Eleuthera - on a side hill near Gregory Town, Coker 367.

New Providence - So far only seen in the Governor's Garden (Britton 153; Millsp. 2247) where it is planted from Harbor Island. Mr. Brace remarks that he once knew a bush of this species in the scrubland back of Nassau but that it has long since disappeared.

Guettarda elliptica, Sw.

In coppices and scrub lands:

Abaco - Cherokee Scund, Brace 1935.

Great Bahama - Eight Mile Rocks, B. \& M. 2583 ; West End, Brace 3554. Andros - Lisbon Creek, Northrop 677.

'Hog Island - B. \& Br. 349 .

New Providence - near Nassau, Brace 166; Hitchcock; Fort Montague, B. \& Br. 173 .

Eleuthera - Governor's Harbor, Hitchcock.

Great Guana Cay - B. \&o $M$.

Exuma - B. \& M. 2979.

Cat Island - Arthur's Town, Coker 425.

Long Island - Clarence Harbor, Coker 515.

Fortune Island - Hitchcock.

Inagua $-N$. \& T. 1266 ; Hitchcock.

Guettarda Krugii Urban.

In scrub lands and rocky coppices:

Man o'War Cay - Brace 1582.

Great Guana Cay - B. \&o M. 2870.

Exuma - scrub lands along Hayne's Road, B. \& M. 3022.

Little Inagua $-N$. \& $T$. 1309 .

Inagua - Mathew Town, N. \& T. 918, 919, 1369; Hitchcock (G. calyptrata).

Grand Turk Island $-N$. \& $T .3800$.

Guettarda scabra Lam.

In scrub lands and coppices:

Abaco - Cherokee Sound, Brace I9og.

Great Bahama - Eight Mile Rocks, B. \& M. 2399.

Andros - at Fresh Creek, Northrop, 535, 730.

New Providence - Near Nassau, Curtiss sine num.; Hitchiock; Brace 186, 197; Village Road and Farringdon Road, B. \& Br. 369, 259.

Eleuthera - opposite Spanish Wells, Coker 318.

Inagua - Hitchcock.

\section{Antirrhoea.}

This genus is being considered by Dr. Britton, 
Laugeria densiflora (Griseb.) Benth. \& Hook.

A viscid shrub frequent in coppices:

Abaco - Marsh Harbor, Brace 1604, 1614, 1714.

New Providence - Eggers 4460; Curtiss sine num.; Brace 380; Blue Hills Road, Coker 551 ; Soldiers Road, B. \& Br. 587; Fox Hills, E. G. B. 3389 ; Hitchcock.

\section{Erithalis fruticosa Linn.}

Everywhere. In low, sandy coppices, sandy thickets, scrub lands, coastal thickets and sand dunes, where it is genérally known as "BLACK TORCH."

Man o'War Cay - Brace 1560.

Abaco - Cherokee Sound, Brace 1899.

Great Bahama - Eight Mile Rocks, B. \& M. 2371 ; West End, Brace 3511 .

Great Sturrup Cay $-B$. \& $M$.

Little Harbor Cay - B. \& M. 2229.

Great Harbor Cay $-B$. \& $M$.

Frozen Cay $-B$. \& $M$.

Whale Cay $-B$. \& $M$.

South Bimini - Millsp. 2413

Gun Cay - Millsp. 2311 .

Andros - Deep Creek and Red Bays, Northrop 601, 365 .

Silver Cay - Millsp. 2449.

Rose Island - B. \& M. 2126.

New Providence - near Nassau, Cooper 4; Brace 27; Coker 73, 161, 192; Wight 138; South Shore, Britton 43,52; Millsp. 2115, 2123 ; Hitchcock.

Eleuthera - Governor's Harbor, Hitchcock.

Exuma Chain - on all cays visited and on Exuma Island, B. \& $M$.

Cat Island - Port Howe, Hitchcock.

Watlings Island - Hitchcock; Coker 463.

Crooked Island - Hitchcock.

Fortune Island - Hitchcock.

Inagua - Hitchcock ; $N$. \& T. 949, 1029, 1054, 1443.

Sheep Cay $-N$. \& T. 1149 .

Grand Turk Island - N. \& T.3768, 3809, 3816, 3817 .

The form of the exposed sea beaches has leaves nearly orbicular, in that of the denser coppices the leaves are quite narrowly lanceolate.

Erithalis odorifera Jacq.

"Common in coppices on New Providence, and distinct from the more abundant E. fruticosa L., being much taller, the corolla twice as 
large as in that species, the fruit and persistent calyx-limb larger."- N. L. Britton.

Andros - Deep Creek, Northrop 739 (E. rotundata).

New Providence - low coppices, Coker 43 (E. fruticosa); near Nassau, $B$. \& Br. 234, Blue Hills road, 580 , Lake Cunningham 605 ; Britton 147; Millsp. 2242 ; Nassau, Curtiss sine num.:

\section{Phialanthus myrtilloides Griseb.}

Coppices and scrublands. Known generally as "CANDlE Wood." Andros - Fresh and Stafford Creeks, Northrop 698, 541.

New Providence - near Nassau, B. \& Br. 677; Curtiss 189.

Watlings Island - Coker 469.

Exuma -- near Rolltown, B. \& M. 3081 .

Fortune Island - Hitchcock.

Little Inagua $-N$. \&. T. 1196.

Inagua $-N$. \& T. 1271, 1379,

Chiococca alba (L.) Rusby.

C. racemosa Jacq. Pine barrens, coppices and scrub lands common : Green Turtle Cay - Brace 1484.

Abaco - Marsh Harbor, Brace 1713, 1789, Butler Bay, 1522, and Eight Mile Bay, 1881 .

Great Bahama - Barnett's Point, B. \& $M .2713$.

Great Harbor Cay - B. \& $M$.

Lignum Vitae Cay - B. \& $M$.

Goat Cay - B. \& $M$.

Mangrove Cay - Bryant 7 .

Silver Cay - Millsp. 2447 .

Rose Island $-B$. \& $M$.

New Providence - near Nassau, Cooper 9; Northrop 28; B. \& Br. 230, 434, 656 ; Wight 149.

Eleuthera - Governor's Harbor, Hitchcock; Gregory Town, Coker 363.

Wide Opening, Cay north of $-B$. \& $M$.

Exuma - B. \& $M$.

Cat Island - Port Howe, Hitchcock. 、

Crooked Island - Hitchcock (C. parvifolia).

Inagua $-N$. \& $T .1298$.

Chiococca parvifolia Wulls.

In coppices, infrequently collected :

Abaco - Marsh Harbor, Brace 1839.

Andros - Deep Creek, Northrop 688. 
New Providence - near Winton, B. \&. Br. 729 ; along Soldiers Road, Coker 557 ; near Nassau, Brace 80.

Chiococca pinetorum Britton sp. nov.

A vine, creeping or low-climbing sometimes $1.5 \mathrm{~m}$. long, usually shorter. Branches short; leaves ovate, lanceolate or elliptic, small, $2-4.5 \mathrm{~cm}$. wide, varying from acute to obtuse at the apex, narrowed at the base, coriaceous, dark green and shining above, paler and dull beneath, the midvein inıpressed on the under side, the lateral veins few and obscure, the petiole slender, $2-3 \mathrm{nmm}$. long; racemes few-flowered, shorter than the leaves or about equalling them; pedicels about as long as the calyx, or sometimes a little longer; calyx campanulate $2 \mathrm{~mm}$. long; corolla funnel-form-campanulate, about $5 \mathrm{~mm}$. long, white or cream color, veined with purple; berry white, somewhat compressed, 4-6 $\mathrm{mm}$. in diameter.

Differs from C. parvifolia Wullsch. by its smaller darker green leaves, smaller pale corolla and less compressed berries. C. pinetorum occurs only, so far as known, on islands inhabited by Pinus bahamensis Griseb., and in association with it.

Abaco - opposite Cherokee Settlement, Brace 1990.

Great Bahama - Eight Mile Rocks, B. \& M. 2408 ; West End, Brace 3563. Andros - near Red Bays, Northrop 477.

New Providence - near Nassau, Northrop 138 (C. parvifolia); Hitchcock (C. parvifolia); $B$. \& $B r .430$ type; Cooper 19, 25 ; Britton 20; Curtiss 159 (C. parvifolia); B. \& M. 2103; Millsp. 2076, 2238; Coker $18,80,163,185$.

\section{Scolosanthus bahamensis Britton.}

Occasional in coppices :

Andros - at Fresh Creek, Northrop 646 (Scolosanthus sp.).

New Providence - coppice on East Road near Nassau, Coker 138; coppice on Soldiers Road, Millsp. 2488; coppice on Village Road, $B$. \& $\mathrm{Br}, 367$ type.

Strumpfia maritima Jacq.

Borders of brackish swamps and on coastal rocks :

Abaco - opposite Cherokee Settlenient, Brace 1981.

Great Bahama - Allen 33.

Whale Cay - $B$. \& $M$.

Joulter's Cays - Millsp. 2300.

Rose Island $-B$. \& $M .2238$.

New Providence - Hitchcock; Cooper sine num.; Coker 59 ; Curtiss 188; Brace 159, 550; southeast shore near Fox Hills, B. \& Br. 550 ; Dix Point, Northrop 15I; Swingate on the shore rocks, Millsp. 2248 ; Wight I16. 
Eleuthera - Governor's Harbor, Hitchcock.

Wide Opening, Cay north of $-B$. \& $M$.

Great Guana Cay - B. \& $M$.

Watlings Island - Hitchcock.

Crooked Island - Hitchcock.

Little Inagua $-N$. \& $T$. $1186,1250$.

Inagua $--N$. \& $T .986$.

Grand Turk Island $-N$. \& $T .3813$; Hjalmarsson.

Psychotria bahamensis Millsp.

Bull. N. Y. Bot. Gard. 3: 45 I. In coppices and thickets. Fruits globular, bright orange; flowers white.

Great Bahama - Eight Mile Rocks, B. \& M. 2529; Brace 3675; Barnett's Point, B: 今 M. 2665.

Great Sturrup Cay - B. \& M. 3260.

Lignum Vitae Cay $-B$. o $M$.

Great Harbor Cay - B. \& $M$.

South Bimini - Millsp. 3478 .

Rose Island - B. \&o M. 2134.

New Providence $-B$. \& Br. 206 type, 208, 662; Earle 43, 52; Britton 17 ; Millsp. 2483 ; E. G. B. 3359.

Fortune Island - Hitchcock (Myrstiphyllum undatum). (Reported on Eleuthera in Pl. Baham p. 94, label says Fortune Is.)

(Psychotria lanceolata Nutt.

This species reported by Grisebach as having been seen in the socalled Swainson collection, has not yet been re-collected in the Bahamas.)

\section{Psychotria ligustrifolia (Northrop)}

Myrstiphyllum ligustrifolium, Northrop, Flora New Prov. \& Andros p. 68.

New Providence -- near Nassau, Northrop 206 type ; Blue Hills, Millsp. 2071 ; Wight 205.

North Cat Cay - Millsp. 2342.

Psychotria pubescens Sw.

In coppices and pine barrens :

Abaco - Marsh Harbor, Brace 1836.

Great Bahama - Eight Mile Rocks, Brace 3700.

Andros - Conch Sound, Northrop, 585 (Myrstiphyllum pubescens).

New. Providence - Farringdon Road, B. \& Br. 204; near Nassau, Cooper 78; Brace 168.

Cat Island - Port Howe, Hitchcock (Myrstiphyllum undatum). 
Psychotria undata Jacq.

In coppices and pine barrens. The common form in the Bahamas.

Known as "WiLd Coffee."

Abaco - Marsh Harbor, Brace 1596, 1613.

Great Bahama -- West End, Brace 3547.

South Bimini - Millsp. 2381 .

Andros - Nichols Town, Northrop 361 (Myrstiphyllum undatum).

Hog Island $-B$. \& Br. 347 .

Rose Island $-B$. \& $M .2116$.

New Providence - Britton 26, 100, 117, 142; Coker 28, 29; B. \& Br. 179,347,437; Millsp. 2051, 2083,2180,2197, 2236, 2484; E. G. B. 3149, 3147; Brace 20; Cooper 59; Hitchcock (Myrstiphyllum undatum).

Eleuthera - opposite Spanish Wells and at Tarpum Bay, Coker 330, 412.

Great Guana Cay - B. \& M. 2868.

Exuma - near Georgetówn, B. \& $M .3138$.

Long Island - Clarence Harbor, Coker 507.

Morinda royoc L.

Scrublands and pine barrens. Known as "RhubarB" and "WiLD MulberRy."

Abaco - Marsh Harbor, Great Cistern, and Butler Bay, Brace 1590, $1763,1517$.

Great Bahama - Eight Mile Rocks, B. \& $M$. 2427; West End, Brace 3487 .

Andros - Conch Sound, Northrop 425.

Eleuthera-- Gregory Town, Coker 379.

\section{Ernodea}

This genus is being considered by Dr. Britton.

Hemidiodia ocimifolia (Willd) Schum.

In water holes. Not before collected on the Islands:

New Providence - water hole on Harold Road, B. \& Br. 532.

\section{Borreria}

This genus is being considered by Dr. Britton.

Spermacoce aspera Aubl.

On waste grounds and in sink holes:

Abaco - Eight Mile Bay, 'Brace 1861 .

Great Bahama - Eight Mile Rocks, B. \& M. 2473. 
New Providence - Farringdon Road and Fort Charlotte, $B$. \& Br. 182, 779; Brace 490; Hitchcock; Wight 32.

Eleuthera - Governor's Harbor, Hitchcock.

Cat Island - Port Howe, Hitchcock.

Inagua $-N$. \& T. 1332, 1377; Hitchcock,

Spermacoce tenuior (L.) Lam.

Only to be found in pot or banana holes:

Abaco - Marsh Harbor, Brace 1640.

Great Bahama - Eight Mile Rocks, B. \&o $M .2475$.

New Providence - South West Point, Northrop 319; near Nassau, Eggers

1441; Earle 18 6; Curtiss 18; Blue Hills road, Millsp. 2471.

Eleuthera - Governor's Harbor, Hitchcock.

Great Guana Cay - B. \& M. 2898.

Inagua $-N$. \& $T$. I4I4; Hitchcock.

Galium hispidulum Mx.

Pinelands infrequent. Identical with the species as found in the southeastern United States.

Elbow Cay - Brace 1694.

Abaco - Marsh Harbor, Brace 1621; Which Point, Coker 563.

Great Bahama - Eight Mile Rocks, B. \& M. 2403.

Andros - Conch Sound, Northrop 523 (Relbunium hypocarpium, this is a high mountain species of Jamaica).

New Providence - near Harold Road, $B$. \& Br. 429; near Nassau, Brace 330; Blue Hills, Millsp. 2062.

\section{VERBENACEÆ.}

Ghinia curassavica (L.)

Ischnia verbenacea D. C. Verbena curassavica Linn. Ghinia verbenacea Sw.

Waste grounds, doubtless an introduced weed :

New Providence - Farringdon Road, $B . \& B$ Br. 183 ; near Nassau, Curtiss sine num.; Brace 192, 432 .

\section{Lantana bahamensis Britton.}

In coppices and scrub lands:

New Providence - near Ft. Montague, $B$. \& $B r .174$ type, 328; near Nassau, Curtiss 109; Coker 126 (L. crocea); Northrop 129 (L. camara); E. G. B. 344I; Hitchcock (L. crocea); Millsp. 2089, 2257; Wight 87, 122.

Andros - Conch Sound and Nichols Town, Northrop 561, 352 (L. crocea); Red Bays, 490 (L. camara). 
Eleuthera - Governor's Harbor, Hitchcock (L. crocea).

Exuma - near Georgetown, B. \& $M .2939$.

Cat Island - Port Howe, Hitchcock (L. crocea).

Lantana balsamifera Britton.

The range of this striking species is as yet rather intermittent. It is found in sand and on whitelands, and is known on. Inagua as "Moujean Tea."

Whale Cay - B. \&o M. 2187.

Eleuthera-Tarpum Bay, Coker 410 (L. involucrata).

Little Inagua $-N$. \& $T$. 1211 type, $1198,1203$.

\section{Lantana camara L.}

So far known only from the following Islands :

Watlings - Hitchicock.

Inagua - Mathew Town, $N$. \& T. 1468.

Long Island - Clarence Harbor, Coker $513^{\circ}$ (L. crocea).

(LANTANa CROcea Jacq.

This species, though reported by all writers on Bahamian plants, has not yet been seen from the islands).

Lantana demutata sp. nov.

- Frutex glabro valido ramoso cortice rugoso. Rami virgatis ascendentis, foliis petiolatis oblongis vel oblanceolatis ad basin attenuatis, obtusis margine revolutis crenato-dentatis pagina utraque minute et strigose pilosis, supra reticulato-rugosis infra pilis densis nervis crassis prominentis. Inflorescentia axillaribus longe pedunculatis pedunculæ filosis ad apice intumescentis capitulae brevis globosis 5-8-floris bractae foliosis ovato-rotundatis crassis. calyce paleaceis coronatis irregulariter dentatis margine ciliatis, corolla tubo longis infundibuliformis aliquid curvatis limbo irregulariter lobatis, lobis superiora erectis rotundatis integris, lateralis parvis irregulariter fisso-dentatis inferiora transverse-ovatis reflexis integris ad basi auriculatis. Fructus globosis carnosis, pyrena profunde et similariter tuber cibariae foveulatis.

A spreading, globose-headed, odorless shrub I. 5 to $2 \mathrm{~m}$. high. Leaves $\mathrm{I}-\mathrm{I} .5 \mathrm{~cm}$. x 6-8 mm., petioles $2 \mathrm{~mm}$., peduncles $2-4 \mathrm{~cm}$. long. Calyx I mm., corolla $3.5 \mathrm{~mm}$. The species approximates L. involucrata, from which it differs, however, in many characters. No counterpart of the specimens could be found in Hb. Kew or British Museum.

In scrublands :

Exuma - near Georgetown, Feb. 26, 1905, B. \& M. 2940 type.

Lantana involucrata Linn.

Common in scrub and open lands. Known as "Wild SAGE." Allen's Cay - Brace 1534. 
Abaco - Cherokee Sound, Brace 1940.

Great Bahama - Eight Mile Rocks, B. \& M. 2444.

Great Sturrup Cay - B. \& $M$.

Lignum Vitae Cay $-B$. \& $M$.

Little and Great Harbor Cays - B. \& $M$.

Frozen Cay $-B$. \& $M$.

Whale Cay - B. \& M. 2188 .

Gun Cay - Millsp. 2306.

South Cat Cay-Millsp. 2422.

Mangrove Cay - Bryant 15.

Rose Island $-B$. \& $M .2127$.

New Providence - near Ft. Montague, Northrop 56; Curtiss sine num.; Britton 51; Earle 45; Coker 21; Brace 70; Hitchcock; Wight 45.

Eleuthera - Governor's Harbor, Hitchcock.

Exuma Chain - on all islands visited except Exuma Island, $B$. \& $M$.; Cave Cay, B. \& $M .2821$.

Cat Island - Port Howe, Hitchcock.

Watlings Island - Hitchcock.

Crooked Island - Hitchcock.

Fortune Island - Hitchoock.

Inagua - Mathew Town, N. \& T. 890, 933; Hitchcock.

Grand Turk Island $-N$. \& $T .3763$; Hjalmarsson.

Lantana odorata Linn.

Doubtless rare on the Bahamian Islands. So far only known by the following specimen :

Green Turtle Cay - Brace 1478.

\section{Lantana ovatifolia Britton.}

A peculiar large and coarse-leaved species, sprawling among shrubs and Pteridium in the pine lands of:

Great Bahama - at Eight Mile Rocks, B. \& M. 2430 type; Brace 3686.

NASHIA gen. nov.

Frutices. Truncus 4-costatus. Cortex roseo-cinereus in laciniae tenuiter exfolientibus. Rami opposito robustis, ramuli ad tuberculae reductus. Foliis ad apicem tuberculae fasciculato vel ad rami oppositi dispositis. Inflorescentia capitellata, rhachi crasso, calyce in annulo reducto corolla tubulo-campanúlatis in aestivo valvato lobis 4 aequaliter magnitudo stamina 4 didymamo. Fructus drupaceus, pyrenae in nuculis osseis coherentibus.

I take great pleasure in dedicating this striking genus to Mr. George Valentine Nash, who, in his energetic field work in the Bahamas, South Florida and Haiti, has rediscovered many little known species and amassed very valuable collections toward a flora of these regions. 
Nashia inaguensis sp. nov.

Frutex citronellae odoratis. Rami crassis oppositis tereto-quadrangularis cortex roseo-cinereis in laminii longitudinale exfoliatis. Folia brevissime petiolatis ad ramuli, baculatum et lanatum in fasciculi dispositis, ovatis vel obovatis vel spathulatis vel ligulatis, obtusis ad basin attenuatis pagina supra valde reticulo-rugosa strigosis infra strigose-tomentosis margine ad siccain revolutis integris. Inflorescentia sessilis, ad media ramulorum dispositis; flores $6-8$ albis cum tubo aurentio-luteus, bractae rutelliformis apiculatıs ciliatis floribus longioribus. Calyce minutis annulatis dentatis ciliatis ad rachi persistentibus, corolla campanulatis lobis aequantis duo exterior magnis ad basin auriculatis apice emarginatis duo interior integris, stigma peltatis post anthesis sagittatis, stylis indusis, filmenta abbreviata tubo tertia demittere insertis. Drupa pyriformis extus carnosis, putamen duris laevis.

A stout, straggling, endemic bush 1.5 to $2 \mathrm{~m}$. high, with strong, stout branches issuing at-right angles. Trunk 4-5 dm. long 3-4 cm. in diameter, leaves $5-8 \times 3-5 \mathrm{~mm}$. ; bracts $2.5 \times 2 \mathrm{~mm}$. Drupes fleshy $3 \times 4$ mm.; nutlets $2.5 \times 3.5 \mathrm{~mm}$. So far only known from:

Inagua - in scrublands back of Mathew Town, Oct. 13, 1904, Nash \& Taylor 1006 type, 1454. A sheet also found in the Hitchcock collection marked "Lantana sp. from Inagua Dec. 3, 1890". Mr. Brace sends a small specimen of the species from a garden in Nassau "where it was planted from Inagua." It is well known to the natives, who use the leaves in decoction as a febrifuge known as "Moujean Tea."

\section{Lippia nodiflora (L.) Michx.}

Open sandy places :

Great Bahama - clearing at Eight Mile Rocks, $B$. \& $M$. 2368; West End,. Bract 3575.

North C'at Cay - Millsp. 2337

Andros - Red Bays, Northrop 481.

Hog Island - Northrop 250.

New Providence - near Nassau, Brace 265; near Grants Town, Coker 280; at Lake Cunningham, Britton 105, Millsp. 2185; Wight 162.

Eleuthera - Governor's Harbor, Hitchcock.

Exuma - near Georgetown, B. \&o $M$.

Cat Island - Port Howe, Hitchcock.

Inagua $-N$. \& $T .1091,1276,1465$.

\section{Lippia reptans H. B. K.}

Shores of the sea or brackish marshes:

New Providence - near Nassau, Northrop 114, (L. canescens); Coker 98, (L. canescens); Hitchcock (L. nodiflora). 
Inagua - Lower Savannah near Blakeville, N. \& T. 1131; Hitchcock (L. nodiflora).

Lippia stoechadifolia (L.) H. B. K.

Borders of fresh water pools or'marshes and in water holes :

New Providence - near Clifton, $B$. \& $B r .745$.

Exuma - in a water hole in palmetto lands back of Georgetown, $B$. \& M. 2964.

Cat Island - near Arthur's Town, Coker 430.

Bouchea prismatica (L.) Ktze.

$B$. Ehrenbergii Cham. In waste grounds :

New Providence - at Fort Charlotte, $B$ \& Br. 782; Braie 371.

Valerianodes fruticosa sp. nov.

Stachytarpheta et Stachytarpha et Abena. \$ Pachyurae: Frutex. Caule erectis duro-lignosis superne ramosis, rami teretis glabriusculis, ramuli subtetragonis; foliis ovato-lancolatis basi contracta in petiolum spurium decurrentibus, apice acutis margine plus minusve patentidentatus, pagina supra nitidis subtus pallidulo-viridis glabriusculis, nervi medius alboviridis, utrinque minute nigro-punctatis. Spices terminalibus cylindraceus brevis tortis, glabris, floribus arrectis demuın rhachi-incrassatae immersis bracteis ovato-lanceolatis calyce subcarinatis margine integris non scariosis, calyce compressis bifidis aequantibus. Fructus obpyriformis manifeste apiculatis, pyrenae jugis pars dorsalibus et citra apicem rugis evidentis anastomosantibus.

A shrub $\mathrm{I}-2 \mathrm{~m}$. high with a strong, erect stem one-half to threefourths of a meter long, with white bark and dense, pinkish wood (in our specimen showing $\mathrm{I} 2$ annular rings). Leaves $4-7 \mathrm{~cm}$. long by $\mathrm{I}-2 \mathrm{~cm}$. broad. Spikes 5-ro $\mathrm{cm}$. long, $4 \mathrm{~mm}$. thick. Pyrenae $6 \mathrm{~mm}$. long, by $2 \mathrm{~mm}$. broad, full twice the dimensions of those of $\mathrm{V}$. jamaicensis to which the species appears nearest related. From that species it also strongly differs in its shrub character, short spikes, and punctate glabrous leaves. In scrublands, infrequent:

Great Bahama - West End, Brace 3574.

Rose Island - Jan. 27, 1905, Britton \& Millspaugh 2125 type.

Wide Opening, Cay north of - from crevices in rocks, B. \& M. 2771 .

\section{Valerianodes jamaicensis (L.) Medic.}

In scrublands and open waste grounds:

Great Bahama - Eight Mile Rocks, B. \& M. 2479.

Great Sturrup Cay $-B$. \& $M$.

Goat Cay - B. \& M. 2300.

Lignum Vitae Cay $-B$. \& $M$. 
Mangrove Cay - Bryant 18.

Rose Island $-B$. \& $M$.

New Providence-near Nassau, Earle 8; Brace 320; Northrop 19 (Abena);

Coker 113. (Abena); Millsp. 2465; Hitchcock (Abena); Wight 85.

Eleuthera - Governor's Harbor, Hitchcock (Abena).

Great Guana Cay $-B$. \& $M$.

Exuma $-B$. \& $M$.

Fortune Island - Hitchcock (Abena).

Inagua - N. \& T. 1068; Hitchcock (Abena).

Grand Turk Island - $N$. \& $T .3870$.

\section{Priva lappulacea (L.) Pers.}

$P$. echinata Juss. Waste places near habitations, doubtless an introduced weed.

Green Turtle Cay - Brace 1482.

New Providence - near Nassau, Curtiss 24; B. \&o Br: 534, 655; Earle 12; Coker 268; Wight 40.

Eleuthera - Governor's Harbor, Hitchcock.

Inagua - Hitchcock.

\section{Citharexylon* $s p$.}

A shrub with obovate short-acuminate acute or emarginate glabrous leaves and paniculate inflorescence.

Inagua - Salt Pond Hill, $N$. \& $T .935$.

The specimens are too incomplete for satisfactory specific determination.

\section{Citharexylon Berterii Spreng.(?)}

In coppices:

Andros - Fresh Creek, Northrop 608.

The specimen under this number in herb. Field Col. Museum possesses either teratological or very immature flowers, hence this determination is rendered somewhat doubtful.

\section{Citharexylon caudatum $\mathrm{L}$.}

In a coastal coppice :

Andros - Couch Sound, Northrop 571 (C. lucidum) Cham. \& Schlecht.

(Citharexylon quadrangulake Jacq.

Prof. Coker's specimens referred to this species in his "Vegetation. The Bahama Islands" have not been seen by the writer.) 


\section{Citharexylon villosum Jacq.}

In coastal coppices:

Abaco - Eight Mile Bay, Brace 1882.

New Providence - at Southwest Bay and along Farringdon Road, $B$. \& Br. 499, 233; near Nassau, Hitchcock.

Cat Island - Port Howe, Hitchcock.

This species shows considerable variation in leaf outline, degree of pubescence and size and color of the pyrenae. Mr. Combs' No. 468 from Cieneguita, Cuba ; Mr. Brace's Abaco No. 1882; and Mr. Nash's 991 from Hayti are broad leaved forms. These pass through Britton \& Brace's New Providence 499 to narrower leaved forms representing C. bahamense Millsp. (Bull. N. Y. Bot. Gard. 3. 450.) To this form are referable, Britton \& Brace's New Providence 233 type; Hitchcock's New Providence and Cat Island specimens; and Paulsen's St. Thomas No. I 56 , the last specimen cited was distributed as C. cinereum L.

\section{Duranta repens Linn.}

D. Plumieri Jacq. Plentiful where found at all. Generally in the pine barrens and open scrublands. Apparently native though possibly introduced and now spreading.

Abaco - Marsh Harbor, Brace 1740.

Great Bahama - pine barrens at Eight Mile Rocks, B. \& M. 2434; Brace 3687.

New Providence - near Nassau, Northrop 39: Cooper 33; Curtiss 1; Coker 61; Brace 22; Millsp. 2050; Hitchcock; Wight 4, 49.

Eleuthera - Governor's Harbor, Hitchcock.

\section{Callicarpa fulva Rich.}

In scrub land and coppiçe:

\section{Cat Island - Hitchcock.}

Mr. Hitchcock's plant, while having (on account of its greatly reduced leaves) a quite distinct general appearance of difference from C. fulva (as well represented by C. Wright's i 357 Monte Verde, Cuba, May 30, I 859,) nevertheless has no other characters of differentiation. It is fairly well connected in leaf size and form with the Wright plant through Mr. Wright's other I 357 collected at the base of Farallones, Sept. 29, 1860, which is in the same fruiting stage as the Hitchcock plant. Of this Farallones plant Mr. Wright says, "A slender bush 6 to ro feet high: in thick woods."

Sauvalle (Fl. Cub. II3) considers this species synonymous with the Jamaican C. ferruginea Swartz, this however is not the case, the differences are broad and evident. 
Grisebach in his Catalogus Plantarum Cubensium includes under C. fulva Rich. Mr. Wright's Majanabajo, Cuba 3I 73 with the remark "forma foliis lanceolatis." The sheet of this number in Herb. Gray, Cambridge (one of Mr. Wright's original series), proves to be an entirely different species. This may be designated as follows:

\section{Callicarpa lancifolia sp. nov.}

Ranulis cum cymis petiolis foliorumque tomento stellari ferrugineo canescentibus, foliis crassis lanceolatis apice acutis ad basin attenuatis brevipetiolatis, supra nigra, ad sicca valde reticulato-rugosis et minute resinosis, infra lutea dense stellato-canescentibus, margine leviter et irregulariter crenato-denticulosis. Cymis axillaribus folio medio brevioribus ramoso-dichotomis, calyce campanulatis dense resino-granulatis cum quidam pilae stellatis et perpauci hirtis, limbo in lobis triangularis valde divisis.

Eastern Cuba, C. Wright No. 3173 in Herb. Gray, Cambridge, U.S. A. type. Leaves $5 \mathrm{~cm}$. long, I $\mathrm{cm}$. broad, strongly bicolor; they differ from those of $\mathrm{C}$. fulva Rich. in their shape, the far less prominence of the veins beneath, and in not having the pubescence thereon so thick and strongly tufted.

\section{Petitia domingensis Jacq.}

P. Poeppigii Schl. In pine barrens and scrublands:

Abaco-Marsh Harbor, Brace 1630, 1721.

Great Bahama - Eight Mile Rocks, B. \& M. 2593.

Andros - Nichols Town, Northrop 358 .

Mangrove Cay - Coker 224.

New Providence - near Nassau, Cooper 34; Eggers 4201; Britton 27; Millsp. 2090; Wight 206; Coker 162, 556; Curtiss 136; Brace 55; near Lake Cunninghain, B. \& Br. 604; Lake Killarney, E. G. B. 3288.

Eleuthera - Governor's Harbor, Hitchcock (P. Poeppigii).

Cat Island - Arthur's Town, Coker 423 (P. Poeppigii).

\section{PSEUDOCARPIDIUM gen nov.}

Arbusculae aut frutices folia simplicia opposita integra aut crenatodentata, flores paniculata. Calyces campanulata 5-dentata aequalia. Corolla tubulosa 5-fida. Stamina 4, didynama exserta, stigma bifida. Drupa 2-pyreneis, pyrene osseis 2 -loculus monospermis in drupa centralia dispositis.

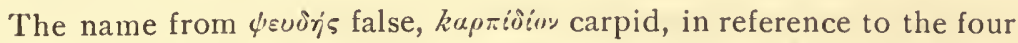
carpid-like prominences on the fruit; these apparent cocci are simply fleshy masses, Richard's fig. 3, pl. 64 in La Sagra to the contrary. notwithstanding. The known species are as follows: 
(Pseudocarpidium ilicifolium (Rich.)

Vitex ilicifolia Rich. in La Sagra Hist. Cuba Bot. 2; I48).

(Pseudocarpidium avicennioides (Rich.).

Vitex avicennioides Rich. in La Sagra Hist. Cuba Bot. 2; 149).

\section{Pseudocarpidium Wrightii sp. nov.}

Frutex a basi ramosissimis, rami glabris crassis, cortice argenteo-albis. Foliis coriaceis late obovatis vel orbiculatis vel ovato-lanceolatis apice obtusis, basi subcordatis, margine superante subsinuoso-dentatis spinosis vel tantummodo spinosis, pagina supra nitidis valde reticulovenosis, infra cum grana resinosa pulverulentibus ad venae minutissime pubescentibus. Inflorescentia paniculatis, paniculae laxis longe et filosae pedunculatis folio plus longioribus supra dichotomis, tota minutissine resinoso-pulverulentibus, bractae linearis. Calyce campanulatis obtuse 5-deltoideo-dentatis, corolla bilabiatis limbo explanatis, labia superiore erectis integris apice deltoideis, labia inferiore in dentibus quatior productis, dentes lateralia ovatis acutis, dentes inferiore deltoideis. Drupa depressa producte in lobis quatior ad centro bipyreneis, pyrenæ osseis hemisphaeris dorso bi-costatis ventro profunde sulcatis.

A tall, lax and woody shrub with opposite, ascending branches and shining holly-like leaves. Leaves $2.5-3.5 \times 1.3^{-2} \mathrm{~cm}$., petioles about $4 \mathrm{~mm}$., the spines confined to the upper half of the leaf. Peduncles 3 . 5-5 cm.

In coppices and scrublands:

Mangrove Cay - Bryant 2.

Andros - at Fresh Creek, Northrop June 6, I89o, no. 625, type; Wight 261 .

The specimens agree with those of Combs, Calicita, Cuba 239, and Shafer, Madruga, Cuba 74. There are two plants of Wright, Cuba orientali bearing his number $42 \mathrm{I}$, one being this species and the other $\mathrm{P}$. ilicifolium, evidently a case of attempted matching of plants before distribution, unfortunately therefore I am compelled to take the later gathered Bahamian plant for the type.

The foliage form and shape is very variable, never however even approaching that of $\mathrm{P}$. ilicifolium, in which the margins are crenate and spinous throughout, while those of P. Wrightii are simply spinous and that only on the upper half of the leaf. Both sides of the leaf in $P$. ilicifolium are pubescent, while in P. Wrightii the upper side is shining and strongly reticulate, while the lower surface is resinous pulverulent.

Clérodendron aculeatum (L.) Griseb.

Rare in collections. Only known by:

New Providence — "Escaped" near Nassau, Northrop 296 (Ovieda). Inagua $-N$. \& $T$. 1471 . 
Ovieda fragrans (Willd.) Hitch.

Escaped from cultivation and becoming fully naturalized along roadways on:

New Providence - Blue Hills Road, Northrop 328; roadside at Lake Cunningham, Millsp. 2221; Nassau, cult. Hitchcock.

Avicennia nitida Jacq.

In salt and brackish borders, nowhere common:

Abaco - Cherokee Sound, Brace 1849.

Great Bahama-Barnett's Point, $B$. \& $M$.

Great Harbor Cay - In the large savannah, $B$. \& $M$.

Frozen Cay - B. \& $M$.

South Bimini - at the south end,' Millsp. 2396.

New Providence - collected by M. A. Howe far out from the south side beach, Millsp. 2482.

Andros - at Mastic Point, Northrop 593 .

Ship Channel Cay - B. \& $M$.

Great Guana Cay - B. \& $M$.

Exuma - in the tide pond at Georgetown, $B$. \& $M$.

Fortune Island - Hitchcock.

Inagua - $N$. \&. $T 1326$.

Grand Turk Island $-N$. \& $T .3823$.

\section{SOLANACE $Æ$.}

Solanum didymacanthum sp. nov.

$\S$ Leptostemonum-Graciliftora. Rami pilis stellatis pilosi, aculiatissimis, foliis minutis integris utrinque stellati-pilosis. Caulis fruticosus ramosissimus $\mathrm{I}-\mathrm{I} .5 \mathrm{~m}$. alt. Rami nigro-viridibus subtomentosis, pilis stellaris, aculeatissimis, aculeis acicularibus ad nodi geminibus foliis bis vel tres longioribus, praegracilis leviter recurvis divaricatohorizontalibus rubro-brunneus basi incrassatis, medio centim. longis. Foliis fasciculatis omnes minutis, petiolatis, oblongis vel obovatis integris $2-2.5 \mathrm{~min}$. longis utrinque stellati-pilosis, basi acutis, apice acutis vel obtusis. Inflorescentia solitaria terminalia. Pedunculi $3 \mathrm{~mm}$. longi, uniflori, stellato-pilosi inermi; calyce stellato-pilosis inermis 5dentatis, laciniis lanceolato-triangularis acutis, I mm. longis; corolla alba (?) 5-partita, laciniis liguliformis stellato-pilosis, $8 \mathrm{~mm}$. longis. Stamina 5 corolla nonnihil breviore. Ovarium globosum minimum; stylus filiformis staminibus longior. Bacca ignota.

A densely virgate-branching, spiny shrub, with minute leaves, the uppermost of which often bear a single spine central upon the midrib of the same length and character as those of the nodes; slightly recurved, needle-like spines full twice or thrice the length of the 
leaves, issuing in twins from each node; and single, comparatively large flowers terminal on each branchlet.

Exuma - in the open scrubland near the west end of Hayne's road, $B$. \& M. 3037 type.

Cat Island - at Port Howe, Hitchcock. This specimen has ovate-spatulate leaves as long as or nearly the length of the spines or sometimes exceeding them. 



